\title{
DISCRETE FUNCTIONAL ANALYSIS TOOLS FOR DISCONTINUOUS GALERKIN METHODS WITH APPLICATION TO THE INCOMPRESSIBLE NAVIER-STOKES EQUATIONS
}

\author{
DANIELE A. DI PIETRO AND ALEXANDRE ERN
}

\begin{abstract}
Two discrete functional analysis tools are established for spaces of piecewise polynomial functions on general meshes: (i) a discrete counterpart of the continuous Sobolev embeddings, in both Hilbertian and non-Hilbertian settings; (ii) a compactness result for bounded sequences in a suitable Discontinuous Galerkin norm, together with a weak convergence property for some discrete gradients. The proofs rely on techniques inspired by the Finite Volume literature, which differ from those commonly used in Finite Element analysis. The discrete functional analysis tools are used to prove the convergence of Discontinuous Galerkin approximations of the steady incompressible Navier-Stokes equations. Two discrete convective trilinear forms are proposed, a nonconservative one relying on Temam's device to control the kinetic energy balance and a conservative one based on a nonstandard modification of the pressure.
\end{abstract}

\section{INTRODUCTION}

Discontinuous Galerkin (DG) methods were introduced over thirty years ago to approximate hyperbolic and elliptic PDEs (see e.g. 2, 17 for an historical perspective), and they have received extensive attention over the last decade. For linear PDEs, the mathematical analysis of such methods is well-understood; see e.g. 2] for a unified analysis for the Poisson problem, [15] for advection-diffusion equations with semidefinite diffusion, and [17, 18, 19] for a unified analysis encompassing hyperbolic and elliptic PDEs in the framework of Friedrichs' systems. The situation is substantially different when dealing with nonlinear second-order PDEs. Indeed, although DG methods have been widely used for such problems, their mathematical analysis has hinged almost exclusively on strong regularity assumptions on the exact solution. This is in stark contrast with the recent literature on Finite Volume (FV) schemes where, following the penetrating works of Eymard, Gallouët, Herbin and co-authors (see e.g. [21, 22, 23]), new discrete functional analysis tools have been derived allowing one to prove the convergence to minimum regularity solutions, i.e. solutions belonging to the natural functional spaces in which the weak formulation of the PDE is set. The key ideas can be summarized as follows:

(i) an a priori estimate on the discrete solution and an associated compactness result are used to infer the strong convergence of a subsequence of discrete solutions to a function $u$ in some Lebesgue space, say $L^{2}(\Omega)$;

Received by the editor May 12, 2008 and, in revised form, March 25, 2009.

2010 Mathematics Subject Classification. Primary 65N12, 65N30, 76D05, 35Q30, 76D07.

(C)2010 American Mathematical Society

Reverts to public domain 28 years from publication 
(ii) the construction of a discrete gradient converging to $\nabla u$ in a suitable Lebesgue space allows one to prove that the limit $u$ actually belongs to some space with additional regularity, say $H_{0}^{1}(\Omega)$;

(iii) the convergence of the scheme is finally proved testing against the projection of a smooth function belonging to some dense subspace, say $C_{c}^{\infty}(\Omega)$.

When the exact solution is unique, the convergence of the whole sequence of discrete approximations is deduced. Moreover, stronger convergence results on the discrete gradient can be derived using the dissipative structure of the problem at hand whenever available.

The present analysis relies on two discrete functional analysis tools in piecewise polynomial spaces on general meshes of a bounded Lipschitz domain $\Omega \subset \mathbb{R}^{d}$ (DG

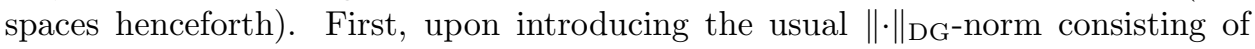
the broken gradient plus a jump term (see (5)) as well as non-Hilbertian variants thereof denoted by $\|\cdot\|_{\mathrm{DG}, p}$ for $1 \leq p<+\infty$ (see (71)), we prove discrete Sobolev embeddings that are the counterpart of those valid at the continuous level,

$$
\left\|v_{h}\right\|_{L^{q}(\Omega)} \leq \sigma_{p, q}\left\|v_{h}\right\|_{\mathrm{DG}, p}, \quad \forall v_{h} \in V_{h}^{k},
$$

for suitable indices $q$ and $p$ and with the DG space $V_{h}^{k}, k \geq 1$, defined by (44). Probably the best known discrete embedding of such a type is the so-called broken Poincaré-Friedrichs inequality obtained with $p=q=2$ and valid more generally on a broken Sobolev space; see e.g. [1, [5]. In the Hilbertian case for the DG norm $(p=2)$, broken Sobolev embeddings have been derived recently by Lasis and Süli [29]. We also refer to Karakashian and Jureidini 27] for the case $q=4$ and $d \in\{2,3\}$ and to Girault, Rivière, and Wheeler [26 for general $q$ and $d=2$. An important point is that the present proofs are substantially different from the ones in the finite element literature, which rely on elliptic regularity or on nonconforming finite element interpolants. Indeed, we take inspiration from the techniques used in 22 in the case of piecewise constant functions. A crucial observation is that the BV norm defined in Lemma 6.2 below is controlled by the $\|\cdot\|_{\mathrm{DG}}$-norm and also by its non-Hilbertian variants. An important advantage is that the present technique of proof incorporates the use of general, nonmatching, polyhedral meshes; that is, under mild assumptions specified below, meshes can possess hanging nodes and consist of elements of various shapes. We only establish the embedding results in DG spaces, and not in the larger setting of broken Sobolev spaces. The latter are indeed not used in the convergence proofs below.

The second functional analysis tool derived herein is a compactness result for bounded sequences in the $\|\cdot\|_{\mathrm{DG}}$-norm and its non-Hilbertian versions. Here again, the proof is quite simple and is inspired from [22]: it consists of using Kolmogorov's Compactness Criterion (see e.g. [7, Theorem IV.25]) based on uniform translate estimates in $L^{1}\left(\mathbb{R}^{d}\right)$ together with the above discrete Sobolev embeddings and a discrete gradient operator that is shown to be weakly convergent in some $L^{p}(\Omega)$ space with $p>1$. Similar results for Sobolev embeddings and compactness of a discrete gradient have been obtained independently by Buffa and Ortner [9].

In the present work we also show how the above analysis tools can be applied to prove the convergence of DG methods under minimal regularity assumptions on the exact solution. In this respect, the weakly consistent discrete gradient operator defined by (12) plays a central role. A further step, going beyond the present scope, could be to consider nonsmooth solutions with localized singularities and 
to derive convergence rates for the error away from these singularities. In the present work, we consider the steady incompressible Navier-Stokes equations as a model problem. Various DG approximations of this problem have been investigated recently [3, 10, 26, 27, 31]. Here, we identify a set of design conditions on the discrete convective trilinear form to prove convergence. Two discrete convective trilinear forms are proposed, a nonconservative one relying on Temam's device to control the kinetic energy balance 33 and a conservative one based on a nonstandard modification of the pressure hinted at in 10.

The paper is organized as follows. 22 introduces the discrete setting, including the assumptions on the meshes, the DG spaces, and the discrete gradient operators, whose weak convergence is proven in Theorem 2.2, \$3 is concerned with the Poisson problem; its purpose is to show how the diffusive term is analyzed. The main result is Theorem 3.1] \$4 deals with the Stokes equations; its purpose is to show how the velocity-pressure coupling is handled. The main result is Theorem 4.1 . $\$ 5$ is concerned with the steady incompressible Navier-Stokes equations; its main result is Theorem 5.1. Finally, $\$ 6$ contains the discrete functional analysis tools in DG spaces. The main results are Theorems 6.1 and 6.3 which are presented in a non-Hilbertian setting since their validity extends beyond the model problems considered in this work.

\section{The Discrete SetTing}

2.1. Meshes. Let $\Omega$ be an open bounded connected subset of $\mathbb{R}^{d}(d>1)$ whose boundary $\partial \Omega$ is a finite union of parts of hyperplanes.

Definition 2.1 (Admissible meshes). Let $\mathcal{H}$ be a countable set. The family $\left\{\mathcal{T}_{h}\right\}_{h \in \mathcal{H}}$ is said to be an admissible mesh family if the following assumptions are satisfied:

(i) for all $h \in \mathcal{H}, \mathcal{T}_{h}$ is a finite family of nonempty connected (possibly nonconvex) open disjoint sets $T$ forming a partition of $\Omega$ and whose boundaries are a finite union of parts of hyperplanes;

(ii) there is a parameter $N_{\partial}$, independent of $h$, such that each $T \in \mathcal{T}_{h}$ has at most $N_{\partial}$ faces. A set $F \subset \partial T$ is said to be a face of $T$ if $F$ is part of a hyperplane, and if either $F=\partial T \cap \partial \Omega$ or there is $T^{\prime} \in \mathcal{T}_{h}, T^{\prime} \neq T$, such that $F=\partial T \cap \partial T^{\prime}$;

(iii) there is a parameter $\varrho_{1}$ independent of $h$ such that for all $T \in \mathcal{T}_{h}$,

$$
\sum_{F \subset \partial T} h_{F}|F| \leq \varrho_{1}|T|
$$

where $h_{F}$ denotes the diameter of the face $F,|F|$ its $(d-1)$-dimensional measure and $|T|$ the $d$-dimensional measure of $T$;

(iv) for all $h \in \mathcal{H}$, each $T \in \mathcal{T}_{h}$ is affine-equivalent to an element belonging to a finite collection of reference elements;

(v) the ratio of the diameter $h_{T}$ of any $T \in \mathcal{T}_{h}$ to the diameter of the largest ball inscribed in $T$ is bounded from above by a parameter $\varrho_{2}$ independent of $h$;

(vi) there is a parameter $\varrho_{3}$, independent of $h$, such that for all $T \in \mathcal{T}_{h}$ and for all faces $F \subset \partial T, h_{F} \geq \varrho_{3} h_{T}$.

For each $h \in \mathcal{H}$, we define $\operatorname{size}\left(\mathcal{T}_{h}\right) \stackrel{\text { def }}{=} \max _{T \in \mathcal{T}_{h}} h_{T}$. The parameters introduced in the above definition will be referred to as the basic mesh parameters and collectively denoted by the symbol $\mathcal{P}$. 
Remark 2.1. Assumptions (v) and (vi) will not be needed in $₫ 6$ to prove the discrete Sobolev embeddings nor the weak convergence of discrete gradients.

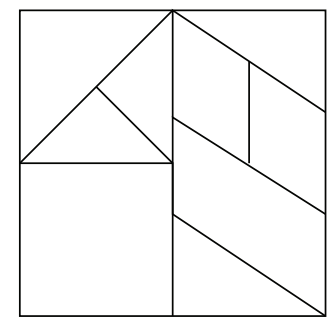

Figure 1. An example of admissible mesh

Figure 1 presents an example of admissible mesh in two space dimensions. The mesh faces are collected in the set $\mathcal{F}_{h}$. The set $\mathcal{F}_{h}$ is partitioned into $\mathcal{F}_{h}^{i} \cup \mathcal{F}_{h}^{b}$, where $\mathcal{F}_{h}^{b}$ collects the faces located on $\partial \Omega$, and $\mathcal{F}_{h}^{i}$ the remaining ones. For $F \in \mathcal{F}_{h}^{i}$, there are $T_{1}$ and $T_{2}$ in $\mathcal{T}_{h}$ such that $F=\partial T_{1} \cap \partial T_{2}$, and we define $\nu_{F}$ as the unit normal vector to $F$ pointing from $T_{1}$ to $T_{2}$. For any function $\varphi$ such that a (possibly two-valued) trace is defined on $F$, let

$$
\llbracket \varphi \rrbracket \stackrel{\text { def }}{=} \varphi_{\mid T_{1}}-\varphi_{\mid T_{2}}, \quad\left\{\{\varphi\} \stackrel{\text { def }}{=} \frac{1}{2}\left(\varphi_{\mid T_{1}}+\varphi_{\mid T_{2}}\right) .\right.
$$

For $F \in \mathcal{F}_{h}^{b}, \nu_{F}$ is defined as the unit outward normal to $\Omega$, while the jump and average are conventionally defined as $\llbracket \varphi \rrbracket \stackrel{\text { def }}{=} \varphi$ and $\{\varphi\} \stackrel{\text { def }}{=} \varphi$.

For any integer $k \geq 0$ and for all $T \in \mathcal{T}_{h}$, let $\mathbb{P}_{k}(T)$ denote the vector space of polynomial functions defined on $T$ with real coefficients and with total degree less than or equal to $k$. Owing to assumptions (iii) and (iv) in Definition 2.1, there is $c_{k, \mathcal{P}}$ such that, for all $h \in \mathcal{H}$ and for all $T \in \mathcal{T}_{h}$,

$$
\forall v_{h} \in \mathbb{P}_{k}(T), \quad \sum_{F \subset \partial T} h_{F} \int_{F}\left|v_{h}\right|^{2} \leq c_{k, \mathcal{P}} \int_{T}\left|v_{h}\right|^{2} .
$$

Here and in what follows, the symbol $c$ will be used to denote a positive generic constant whose value can change at each occurrence. To keep track of the dependency of such constants on some parameters, subscripts will be used whenever relevant.

2.2. DG spaces. Let $k \geq 0$ and consider the finite dimensional space

$$
V_{h}^{k} \stackrel{\text { def }}{=}\left\{v_{h} \in L^{2}(\Omega) ; \forall T \in \mathcal{T}_{h}, v_{h \mid T} \in \mathbb{P}_{k}(T)\right\} .
$$

For $k \geq 1$, this space is equipped with the norm

$$
\left\|v_{h}\right\|_{\mathrm{DG}}^{2} \stackrel{\text { def }}{=} \sum_{T \in \mathcal{T}_{h}} \int_{T}\left|\nabla v_{h}\right|^{2}+\sum_{F \in \mathcal{F}_{h}} \frac{1}{h_{F}} \int_{F}\left|\llbracket v_{h} \rrbracket\right|^{2},
$$

where $|\cdot|$ denotes the Euclidean norm in $\mathbb{R}^{d}$. For further use, it will be convenient to introduce the seminorms

$$
\left|v_{h}\right|_{\mathrm{J}, \mathcal{F}, \pm 1}^{2} \stackrel{\text { def }}{=} \sum_{F \in \mathcal{F}} h_{F}^{ \pm 1} \int_{F}\left|\llbracket v_{h} \rrbracket\right|^{2}
$$


where $\mathcal{F}$ is a subset of $\mathcal{F}_{h}$ that will usually be taken equal to $\mathcal{F}_{h}$ or to $\mathcal{F}_{h}^{i}$. Moreover, we define $\nabla_{h} v_{h}$ as the piecewise gradient of $v_{h} \in V_{h}^{k}$, i.e., $\nabla_{h} v_{h} \in\left[V_{h}^{k-1}\right]^{d}$ is such that for all $T \in \mathcal{T}_{h}, \nabla_{h} v_{h \mid T}=\nabla\left(v_{h \mid T}\right)$, so that

$$
\left\|v_{h}\right\|_{\mathrm{DG}}^{2}=\left\|\nabla_{h} v_{h}\right\|_{L^{2}(\Omega)^{d}}^{2}+\left|v_{h}\right|_{\mathrm{J}, \mathcal{F}_{h},-1}^{2} .
$$

The above norms and seminorms can be extended to $C_{c}^{\infty}(\Omega)+V_{h}^{k}$ (larger spaces are not needed henceforth).

A straightforward but important result concerns the approximability of smooth functions in the $\|\cdot\|_{\mathrm{DG}}$-norm. For all $l \geq 0$, let $\pi_{h}^{l}$ denote the $L^{2}(\Omega)$-orthogonal projection from $L^{2}(\Omega)$ onto $V_{h}^{l}$. These projectors will also be applied componentwise to vector-valued functions. Let $\varphi \in C_{c}^{\infty}(\Omega)$. Then, owing to assumptions (iii)-(v) in Definition 2.1, it is clear using classical approximation properties (see e.g. [6, 16]) that, for all $l \geq 1$,

$$
\left\|\varphi-\pi_{h}^{l} \varphi\right\|_{\mathrm{DG}} \rightarrow 0 \quad \text { as } \operatorname{size}\left(\mathcal{T}_{h}\right) \rightarrow 0 .
$$

In what follows, we shall make frequent use of the projector $\pi_{h}^{1}$ which will be simply denoted by $\pi_{h}$.

For ease of exposition, we state Theorem 6.1 in the present Hilbertian setting for the DG norm. A general proof is given in $\$ 6$.

Theorem 2.1 (Discrete Sobolev embeddings). For all $q$ such that

(i) $1 \leq q \leq \frac{2 d}{d-2}$ if $d \geq 3$,

(ii) $1 \leq q<+\infty$ if $d=2$,

there is $\sigma_{q}$ such that

$$
\forall v_{h} \in V_{h}^{k}, \quad\left\|v_{h}\right\|_{L^{q}(\Omega)} \leq \sigma_{q}\left\|v_{h}\right\|_{\mathrm{DG}} .
$$

The constant $\sigma_{q}$ additionally depends on $k,|\Omega|$, and $\mathcal{P}$.

2.3. Discrete gradient operators. For all $F \in \mathcal{F}_{h}$, let $r_{F}^{l}: L^{2}(F) \rightarrow\left[V_{h}^{l}\right]^{d}, l \geq 0$, be the lifting operator defined as follows: For all $\phi \in L^{2}(F)$,

$$
\forall \tau_{h} \in\left[V_{h}^{l}\right]^{d}, \quad \int_{\Omega} r_{F}^{l}(\phi) \cdot \tau_{h}=\int_{F}\left\{\left\{\tau_{h}\right\}\right] \cdot \nu_{F} \phi .
$$

Clearly, the support of $r_{F}^{l}(\phi)$ consists of the one or two mesh elements of which $F$ is a face. Now let $k \geq 1$. For $v_{h} \in V_{h}^{k}$, define

$$
R_{h}^{l}\left(\llbracket v_{h} \rrbracket\right) \stackrel{\text { def }}{=} \sum_{F \in \mathcal{F}_{h}} r_{F}^{l}\left(\llbracket v_{h} \rrbracket\right)
$$

The following discrete gradient operators $G_{h}^{l}: V_{h}^{k} \rightarrow\left[V_{h}^{\max (k-1, l)}\right]^{d}$ will play an important role in the analysis:

$$
\forall v_{h} \in V_{h}^{k}, \quad G_{h}^{l}\left(v_{h}\right) \stackrel{\text { def }}{=} \nabla_{h} v_{h}-R_{h}^{l}\left(\llbracket v_{h} \rrbracket\right)=\nabla_{h} v_{h}-\sum_{F \in \mathcal{F}_{h}} r_{F}^{l}\left(\llbracket v_{h} \rrbracket\right) .
$$

For a given $k \geq 1$, the most natural value for $l$ is $k$ or $(k-1)$, but the values $l=0$ and $l=2 k$ will also be used. It is straightforward to verify, using assumption (ii) in Definition 2.1 that for all $v_{h} \in V_{h}^{k}$,

$$
\left\|R_{h}^{l}\left(\llbracket v_{h} \rrbracket\right)\right\|_{L^{2}(\Omega)^{d}}^{2} \leq N_{\partial} \sum_{F \in \mathcal{F}_{h}}\left\|r_{F}^{l}\left(\llbracket v_{h} \rrbracket\right)\right\|_{L^{2}(\Omega)^{d}}^{2} .
$$


Furthermore, owing to the trace inequality (3) and proceeding as in [8], it is inferred that for all $F \in \mathcal{F}_{h}$,

$$
\left\|r_{F}^{l}\left(\llbracket v_{h} \rrbracket\right)\right\|_{L^{2}(\Omega)^{d}}^{2} \leq c_{k, l, \mathcal{P}} \frac{1}{h_{F}} \int_{F}\left|\llbracket v_{h} \rrbracket\right|^{2} .
$$

As a result,

$$
\left\|R_{h}^{l}\left(\llbracket v_{h} \rrbracket\right)\right\|_{L^{2}(\Omega)^{d}} \leq c_{k, l, \mathcal{P}}\left|v_{h}\right|_{\mathrm{J}, \mathcal{F}_{h},-1} .
$$

Proposition 2.1 (Stability of discrete gradients). Let $k \geq 1$ and let $l \geq 0$. Then,

$$
\forall v_{h} \in V_{h}^{k}, \quad\left\|G_{h}^{l}\left(v_{h}\right)\right\|_{L^{2}(\Omega)^{d}} \leq c_{k, l, \mathcal{P}}\left\|v_{h}\right\|_{\mathrm{DG}} .
$$

Proof. Use the triangle inequality.

Proposition 2.2 (Strong convergence of discrete gradients for smooth functions). Let $k \geq 1$ and let $l \geq 0$. For all $\varphi \in C_{c}^{\infty}(\Omega), G_{h}^{l}\left(\pi_{h} \varphi\right) \rightarrow \nabla \varphi$ in $L^{2}(\Omega)^{d}$.

Proof. Observe that $\left\|G_{h}^{l}\left(\pi_{h} \varphi\right)-\nabla \varphi\right\|_{L^{2}(\Omega)^{d}} \leq c_{l, \mathcal{P}}\left\|\varphi-\pi_{h} \varphi\right\|_{\mathrm{DG}}$ and use (8).

The main property of the discrete gradient operators defined by (12) is their weak convergence in $L^{2}(\Omega)^{d}$ when evaluated on bounded sequences in the $\|\cdot\|_{\mathrm{DG}}$-norm.

Theorem 2.2 (Compactness and weak convergence of discrete gradients). Let $k \geq$ 1. Let $\left\{v_{h}\right\}_{h \in \mathcal{H}}$ be a sequence in $V_{h}^{k}$. Assume that this sequence is bounded in the $\|\cdot\|_{\mathrm{DG}}-$ norm. Then, there exists a function $v \in H_{0}^{1}(\Omega)$ such that as $\operatorname{size}\left(\mathcal{T}_{h}\right) \rightarrow 0$, up to a subsequence, $v_{h} \rightarrow v$ strongly in $L^{2}(\Omega)$ and for all $l \geq 0, G_{h}^{l}\left(v_{h}\right) \rightarrow \nabla v$ weakly in $L^{2}(\Omega)^{d}$.

Proof. Owing to Theorem 6.2 applied with $p=2$ and extending the functions $v_{h}$ by zero outside $\Omega$, there exists a function $v \in L^{2}\left(\mathbb{R}^{d}\right)$ such that as $\operatorname{size}\left(\mathcal{T}_{h}\right) \rightarrow 0$, up to a subsequence, $v_{h} \rightarrow v$ strongly in $L^{2}\left(\mathbb{R}^{d}\right)$. Moreover, since for all $l \geq 0,\left\{G_{h}^{l}\left(v_{h}\right)\right\}_{h \in \mathcal{H}}$ is bounded in $L^{2}\left(\mathbb{R}^{d}\right)^{d}$ owing to Proposition 2.1, up to a new subsequence, there is $w \in L^{2}\left(\mathbb{R}^{d}\right)^{d}$ s.t. $G_{h}^{l}\left(v_{h}\right) \rightarrow w$ weakly in $L^{2}\left(\mathbb{R}^{d}\right)^{d}$. To prove that $w=\nabla v$, let $\varphi \in C_{c}^{\infty}\left(\mathbb{R}^{d}\right)^{d}$ and observe that

$$
\begin{aligned}
\int_{\mathbb{R}^{d}} G_{h}^{l}\left(v_{h}\right) \cdot \varphi= & -\int_{\mathbb{R}^{d}} v_{h}(\nabla \cdot \varphi)-\int_{\mathbb{R}^{d}} R_{h}^{l}\left(\llbracket v_{h} \rrbracket\right) \cdot\left(\varphi-\pi_{h}^{0} \varphi\right) \\
& \left.+\sum_{F \in \mathcal{F}_{h}} \int_{F}\left\{\varphi-\pi_{h}^{0} \varphi\right\}\right] \cdot \nu_{F} \llbracket v_{h} \rrbracket=T_{1}+T_{2}+T_{3} .
\end{aligned}
$$

Letting $\operatorname{size}\left(\mathcal{T}_{h}\right) \rightarrow 0$, we observe that $T_{1} \rightarrow-\int_{\mathbb{R}^{d}} v(\nabla \cdot \varphi)$ and that $T_{2} \rightarrow 0$ since $\left\|\varphi-\pi_{h}^{0} \varphi\right\|_{L^{2}\left(\mathbb{R}^{d}\right)^{d}} \rightarrow 0$ and $\left\{R_{h}^{l}\left(\llbracket v_{h} \rrbracket\right)\right\}_{h \in \mathcal{H}}$ is bounded in $L^{2}\left(\mathbb{R}^{d}\right)^{d}$. Furthermore, the Cauchy-Schwarz inequality, together with assumption (iii) in Definition 2.1 . yields

$$
\left|T_{3}\right| \leq C\left\|\varphi-\pi_{h}^{0} \varphi\right\|_{L^{\infty}\left(\mathbb{R}^{d}\right)^{d}}\left|v_{h}\right|_{\mathrm{J}, \mathcal{F}_{h},-1} \leq C^{\prime}\left\|\varphi-\pi_{h}^{0} \varphi\right\|_{L^{\infty}\left(\mathbb{R}^{d}\right)^{d}}
$$

which tends to zero as $\operatorname{size}\left(\mathcal{T}_{h}\right) \rightarrow 0$. As a result,

$$
\int_{\mathbb{R}^{d}} w \cdot \varphi=\lim _{\operatorname{size}\left(\mathcal{T}_{h}\right) \rightarrow 0} \int_{\mathbb{R}^{d}} G_{h}^{l}\left(v_{h}\right) \cdot \varphi=-\int_{\mathbb{R}^{d}} v(\nabla \cdot \varphi),
$$

implying that $w=\nabla v$. Hence, $v \in H^{1}\left(\mathbb{R}^{d}\right)$ and since $v$ is zero outside $\Omega, v$ is in $H_{0}^{1}(\Omega)$. 
It is useful to introduce for all $l \geq 0$, further discrete gradient operators $\mathcal{G}_{h}^{l}$ : $V_{h}^{k} \rightarrow\left[V_{h}^{\max (k-1, l)}\right]^{d}$ s.t.

$$
\forall v_{h} \in V_{h}^{k}, \quad \mathcal{G}_{h}^{l}\left(v_{h}\right) \stackrel{\text { def }}{=} \nabla_{h} v_{h}-\sum_{F \in \mathcal{F}_{h}^{i}} r_{F}^{l}\left(\llbracket v_{h} \rrbracket\right) .
$$

The difference with respect to the discrete gradient operator $G_{h}^{l}$ defined by (12) is that boundary faces are not included in (17). The discrete gradient operator $\mathcal{G}_{h}^{l}$ also satisfies the conclusions of Propositions 2.1 and 2.2. More importantly, it also satisfies the conclusions of Theorem 2.2. This is so because $\varphi$ in the above proof is compactly supported; hence, as $\operatorname{size}\left(\mathcal{T}_{h}\right) \rightarrow 0$, the mesh becomes fine enough so that all the mesh elements having a boundary face are located outside the support of $\varphi$.

\section{The Poisson PRoblem}

Let $f \in L^{r}(\Omega)$ with $r=\frac{2 d}{d+2}$ if $d \geq 3$ and $r>1$ if $d=2$. Set $r^{\prime} \stackrel{\text { def }}{=} \frac{r}{r-1}$. Consider the following model problem

$$
\begin{cases}-\Delta u=f, & \text { in } \Omega, \\ u=0, & \text { on } \partial \Omega .\end{cases}
$$

The weak formulation of this problem consists of finding $u \in H_{0}^{1}(\Omega)$ s.t. for all $v \in H_{0}^{1}(\Omega)$,

$$
\int_{\Omega} \nabla u \cdot \nabla v=\int_{\Omega} f v
$$

It is well known that this problem is well-posed. In particular, owing to the Sobolev embedding $\|v\|_{L^{r^{\prime}}(\Omega)} \leq \mathcal{S}_{2, r^{\prime}}\|\nabla v\|_{L^{2}(\Omega)^{d}}$ valid for all $v \in H_{0}^{1}(\Omega)$, and using Hölder's inequality, it is inferred that

$$
\|\nabla u\|_{L^{2}(\Omega)^{d}}^{2}=\int_{\Omega} f u \leq\|f\|_{L^{r}(\Omega)}\|u\|_{L^{r^{\prime}(\Omega)}} \leq \mathcal{S}_{2, r^{\prime}}\|f\|_{L^{r}(\Omega)}\|\nabla u\|_{L^{2}(\Omega)^{d}},
$$

yielding the a priori bound $\|\nabla u\|_{L^{2}(\Omega)^{d}} \leq \mathcal{S}_{2, r^{\prime}}\|f\|_{L^{r}(\Omega)}$.

3.1. Symmetric formulations. Let $k \geq 1$. For the sake of simplicity, discrete gradients are built using the lifting operators $r_{F}^{k}$ (see Remark 3.2 below for further discussion) and to alleviate the notation, the superscript $k$ is omitted. This convention is kept for the rest of this work. For all $\left(v_{h}, w_{h}\right) \in V_{h}^{k} \times V_{h}^{k}$, consider the following symmetric DG bilinear form

$$
a_{h}\left(v_{h}, w_{h}\right) \stackrel{\text { def }}{=} \int_{\Omega} G_{h}\left(v_{h}\right) \cdot G_{h}\left(w_{h}\right)+j_{h}\left(v_{h}, w_{h}\right),
$$

with the stabilization bilinear form

$$
j_{h}\left(v_{h}, w_{h}\right) \stackrel{\text { def }}{=} \sum_{F \in \mathcal{F}_{h}} \eta \int_{\Omega} r_{F}\left(\llbracket v_{h} \rrbracket\right) \cdot r_{F}\left(\llbracket w_{h} \rrbracket\right)-\int_{\Omega} R_{h}\left(\llbracket v_{h} \rrbracket\right) \cdot R_{h}\left(\llbracket w_{h} \rrbracket\right),
$$

where $\eta \in \mathbb{R}_{+}$is a penalty parameter. Henceforth, we assume that

$$
\eta>N_{\partial} \text {. }
$$

Observe that for all $v_{h} \in V_{h}^{k}$, (13) yields

$$
\left\|G_{h}\left(v_{h}\right)\right\|_{L^{2}(\Omega)^{d}}^{2}+\left(\eta-N_{\partial}\right) \sum_{F \in \mathcal{F}_{h}}\left\|r_{F}\left(\llbracket v_{h} \rrbracket\right)\right\|_{L^{2}(\Omega)^{d}}^{2} \leq a_{h}\left(v_{h}, v_{h}\right) .
$$


Remark 3.1. The bilinear forms $a_{h}$ and $j_{h}$ can also be written without using lifting operators. We use explicitly the discrete gradient operator $G_{h}$ since it plays a central role in the convergence proof. A straightforward calculation shows that

$$
\begin{aligned}
a_{h}\left(v_{h}, w_{h}\right)= & \int_{\Omega} \nabla_{h} v_{h} \cdot \nabla_{h} w_{h}-\sum_{F \in \mathcal{F}_{h}} \int_{F}\left(\nu_{F} \cdot\left\{\left\{\nabla_{h} v_{h}\right\} \llbracket w_{h} \rrbracket+\nu_{F} \cdot\left\{\nabla_{h} w_{h}\right\}\right\} \llbracket v_{h} \rrbracket\right) \\
& +\sum_{F \in \mathcal{F}_{h}} \eta \int_{\Omega} r_{F}\left(\llbracket v_{h} \rrbracket\right) \cdot r_{F}\left(\llbracket w_{h} \rrbracket\right),
\end{aligned}
$$

yielding the IP-type method introduced in [4]. Other stabilizations are possible. In particular,

$$
j_{h}^{\mathrm{SIPG}}\left(v_{h}, w_{h}\right) \stackrel{\text { def }}{=} \sum_{F \in \mathcal{F}_{h}} \eta \frac{1}{h_{F}} \int_{F} \llbracket v_{h} \rrbracket \llbracket w_{h} \rrbracket-\int_{\Omega} R_{h}\left(\llbracket v_{h} \rrbracket\right) \cdot R_{h}\left(\llbracket w_{h} \rrbracket\right),
$$

yielding the usual Symmetric Interior Penalty method (SIPG) [1]. In this case, the minimal threshold for the penalty parameter $\eta$ depends on the constant in the trace inequality (3). It is also possible to consider the stabilization

$$
j_{h}^{\mathrm{LDG}}\left(v_{h}, w_{h}\right) \stackrel{\text { def }}{=} \sum_{F \in \mathcal{F}_{h}} \eta \frac{1}{h_{F}} \int_{F} \llbracket v_{h} \rrbracket \llbracket w_{h} \rrbracket,
$$

yielding one version of the Local Discontinuous Galerkin method (LDG) [12. The advantage is that the parameter $\eta$ needs only to be positive, but the stencil is enlarged to neighbors of neighbors. Moreover, working with any of the two above stabilization bilinear forms allows one to omit assumption (vi) in Definition 2.1.

Lemma 3.1 (Coercivity). There is $\alpha>0$, depending on $\eta, k$, and $\mathcal{P}$ such that for all $v_{h} \in V_{h}^{k}$,

$$
\alpha\left\|v_{h}\right\|_{\mathrm{DG}}^{2} \leq a_{h}\left(v_{h}, v_{h}\right) .
$$

Proof. Proceeding as in 8 using assumptions (iv) and (vi) in Definition 2.1 yields for all $F \in \mathcal{F}_{h}$,

$$
\frac{1}{h_{F}} \int_{F}\left|\llbracket v_{h} \rrbracket\right|^{2} \leq c_{k, \mathcal{P}}^{\prime}\left\|r_{F}\left(\llbracket v_{h} \rrbracket\right)\right\|_{L^{2}(\Omega)^{d}}^{2} .
$$

Using the triangle inequality, it is then inferred that

$$
\begin{aligned}
\left\|v_{h}\right\|_{\mathrm{DG}}^{2} & \leq 2\left\|G_{h}\left(v_{h}\right)\right\|_{L^{2}(\Omega)^{d}}^{2}+2\left\|R_{h}\left(\llbracket v_{h} \rrbracket\right)\right\|_{L^{2}(\Omega)^{d}}^{2}+\left|v_{h}\right|_{\mathrm{J}, \mathcal{F}_{h},-1}^{2} \\
& \leq 2\left\|G_{h}\left(v_{h}\right)\right\|_{L^{2}(\Omega)^{d}}^{2}+\left(2 N_{\partial}+c_{k, \mathcal{P}}^{\prime}\right) \sum_{F \in \mathcal{F}_{h}}\left\|r_{F}\left(\llbracket v_{h} \rrbracket\right)\right\|_{L^{2}(\Omega)^{d}}^{2} \\
& \leq \max \left(2,\left(2 N_{\partial}+c_{k, \mathcal{P}}^{\prime}\right)\left(\eta-N_{\partial}\right)^{-1}\right) a_{h}\left(v_{h}, v_{h}\right),
\end{aligned}
$$

the last inequality resulting from (24).

Remark 3.2. Coercivity also holds if the stabilization bilinear form $j_{h}$ is defined using liftings of degree $<k$. In this case, the upper bound in (27) also contains the $L^{2}(\Omega)^{d}$-norm of the broken gradient.

For all $h \in \mathcal{H}$, Lemma 3.1 implies that there is a unique $u_{h} \in V_{h}^{k}$ s.t.

$$
a_{h}\left(u_{h}, v_{h}\right)=\int_{\Omega} f v_{h}, \quad \forall v_{h} \in V_{h}^{k} .
$$


Theorem 3.1 (Convergence for the Poisson problem). Let $\left\{u_{h}\right\}_{h \in \mathcal{H}}$ be the sequence of approximate solutions generated by solving the discrete problems (28) on the admissible meshes $\left\{\mathcal{T}_{h}\right\}_{h \in \mathcal{H}}$. Then, as $\operatorname{size}\left(\mathcal{T}_{h}\right) \rightarrow 0$,

$$
\begin{aligned}
u_{h} & \rightarrow u, \quad \text { in } L^{2}(\Omega), \\
\nabla_{h} u_{h} & \rightarrow \nabla u, \quad \text { in } L^{2}(\Omega)^{d}, \\
\left|u_{h}\right|_{\mathrm{J}, \mathcal{F}_{h},-1} & \rightarrow 0,
\end{aligned}
$$

where $u \in H_{0}^{1}(\Omega)$ is the unique solution to (18).

Proof. (i) A priori estimate. Using Lemma 3.1 and Hölder's inequality, it is inferred that

$$
\alpha\left\|u_{h}\right\|_{\mathrm{DG}}^{2} \leq a\left(u_{h}, u_{h}\right)=\int_{\Omega} f u_{h} \leq\|f\|_{L^{r}(\Omega)}\left\|u_{h}\right\|_{L^{r^{\prime}}(\Omega)} .
$$

Hence, owing to Theorem 2.1, the sequence $\left\{u_{h}\right\}_{h \in \mathcal{H}}$ is bounded in the $\|\cdot\|_{\mathrm{DG}}$-norm.

(ii) $L^{2}$-convergence of a subsequence, regularity of the limit and weak convergence of discrete gradient. Owing to Theorem 2.2, there exists $u \in H_{0}^{1}(\Omega)$ such that, as $\operatorname{size}\left(\mathcal{T}_{h}\right) \rightarrow 0$, up to a subsequence, $u_{h} \rightarrow u$ strongly in $L^{2}(\Omega)$ and $G_{h}\left(u_{h}\right) \rightarrow \nabla u$ weakly in $L^{2}(\Omega)^{d}$.

(iii) Identification of $u$ and convergence of the whole sequence. Let us first prove that for all $\varphi \in C_{c}^{\infty}(\Omega)$,

$$
a_{h}\left(u_{h}, \pi_{h} \varphi\right) \rightarrow \int_{\Omega} \nabla u \cdot \nabla \varphi
$$

Indeed, observe that

$$
a_{h}\left(u_{h}, \pi_{h} \varphi\right)=\int_{\Omega} G_{h}\left(u_{h}\right) \cdot G_{h}\left(\pi_{h} \varphi\right)+j_{h}\left(u_{h}, \pi_{h} \varphi\right)=T_{1}+T_{2} .
$$

Clearly, $T_{1} \rightarrow \int_{\Omega} \nabla u \cdot \nabla \varphi$ owing to the weak convergence of $G_{h}\left(u_{h}\right)$ and the strong convergence of $G_{h}\left(\pi_{h} \varphi\right)$ (see Proposition 2.2). Furthermore, $T_{2} \rightarrow 0$ since $T_{2}$ is controlled by $\left|u_{h}\right|_{\mathrm{J}, \mathcal{F}_{h},-1}\left|\pi_{h} \varphi\right|_{\mathrm{J}, \mathcal{F}_{h},-1}$ where the first factor is bounded and the second tends to zero. A direct consequence of (32) is that for all $\varphi \in C_{c}^{\infty}(\Omega)$,

$$
\int_{\Omega} f \varphi \leftarrow \int_{\Omega} f \pi_{h} \varphi=a_{h}\left(u_{h}, \pi_{h} \varphi\right) \rightarrow \int_{\Omega} \nabla u \cdot \nabla \varphi
$$

Thus, $u$ solves the Poisson problem by density of $C_{c}^{\infty}(\Omega)$ in $H_{0}^{1}(\Omega)$. Since the solution of this problem is unique, the whole sequence $\left\{u_{h}\right\}_{h \in \mathcal{H}}$ strongly converges to $u$ in $L^{2}(\Omega)$ and $\left\{G_{h}\left(u_{h}\right)\right\}_{h \in \mathcal{H}}$ weakly converges to $\nabla u$ in $L^{2}(\Omega)^{d}$.

(iv) Strong convergence of the discrete gradient and of the jumps. Owing to (24) and to weak convergence,

$$
\liminf a_{h}\left(u_{h}, u_{h}\right) \geq \liminf \left\|G_{h}\left(u_{h}\right)\right\|_{L^{2}(\Omega)^{d}}^{2} \geq\|\nabla u\|_{L^{2}(\Omega)^{d}}^{2} .
$$

Furthermore, still owing to (24),

yielding

$$
\left\|G_{h}\left(u_{h}\right)\right\|_{L^{2}(\Omega)^{d}}^{2} \leq a_{h}\left(u_{h}, u_{h}\right)=\int_{\Omega} f u_{h},
$$

$$
\begin{aligned}
\limsup \left\|G_{h}\left(u_{h}\right)\right\|_{L^{2}(\Omega)^{d}}^{2} & \leq \limsup a_{h}\left(u_{h}, u_{h}\right) \\
& =\limsup \int_{\Omega} f u_{h}=\int_{\Omega} f u=\|\nabla u\|_{L^{2}(\Omega)^{d}}^{2}
\end{aligned}
$$


Thus, $\left\|G_{h}\left(u_{h}\right)\right\|_{L^{2}(\Omega)^{d}} \rightarrow\|\nabla u\|_{L^{2}(\Omega)^{d}}$, classically yielding the strong convergence of the discrete gradient in $L^{2}(\Omega)^{d}$. Note that $a_{h}\left(u_{h}, u_{h}\right) \rightarrow\|\nabla u\|_{L^{2}(\Omega)^{d}}^{2}$ also. Finally, owing to (24),

$$
\left(\eta-N_{\partial}\right) \sum_{F \in \mathcal{F}_{h}}\left\|r_{F}\left(\llbracket u_{h} \rrbracket\right)\right\|_{L^{2}(\Omega)^{d}}^{2} \leq a_{h}\left(u_{h}, u_{h}\right)-\left\|G_{h}\left(u_{h}\right)\right\|_{L^{2}(\Omega)^{d}}^{2},
$$

and since $\eta>N_{\partial}$ and the right-hand side tends to zero, it is inferred using (27) that $\left|u_{h}\right|_{\mathrm{J}, \mathcal{F}_{h},-1} \rightarrow 0$. Moreover, using (13) to estimate the second term yields

$$
\left\|\nabla_{h} u_{h}-\nabla u\right\|_{L^{2}(\Omega)^{d}} \leq\left\|G_{h}\left(u_{h}\right)-\nabla u\right\|_{L^{2}(\Omega)^{d}}+\left\|R_{h}\left(u_{h}\right)\right\|_{L^{2}(\Omega)^{d}} \rightarrow 0,
$$

as $\operatorname{size}\left(\mathcal{T}_{h}\right) \rightarrow 0$, concluding the proof.

Remark 3.3. We emphasize that the discrete bilinear forms $a_{h}$ and $j_{h}$ are only defined at the discrete level. Thus, the consistency of the method is expressed by the property (32), and not by inserting the exact solution into the bilinear form as is the case in the usual finite element analysis. To proceed in the usual way, the bilinear forms $a_{h}$ and $j_{h}$ must first be extended to a larger functional space, and the two strictly equivalent expressions for $a_{h}$ at the discrete level, namely (21) and (25), do not lead to the same extension, and only the form using discrete gradients can be extended up to $H^{1}(\Omega)$. If the discrete gradients are kept, the extended bilinear form is weakly consistent since for $u$ smooth enough and for all $v_{h} \in V_{h}^{k}$,

$$
a_{h}\left(u_{h}-u, v_{h}\right)=\sum_{F \in \mathcal{F}_{h}} \int_{F} \nu_{F} \cdot\left\{\left\{\pi_{h}^{k}(\nabla u)-\nabla u\right\}\right\} \llbracket v_{h} \rrbracket .
$$

If the equivalent expression (25) is used, the extended bilinear form is strongly consistent if the exact solution is in $H^{3 / 2+\epsilon}\left(\mathcal{T}_{h}\right), \epsilon>0$. In both cases, standard finite element techniques lead to the optimally convergent error bound $\left\|u-u_{h}\right\|_{\mathrm{DG}} \leq$ $c_{u} \operatorname{size}\left(\mathcal{T}_{h}\right)^{k}$ when $u \in H^{k+1}\left(\mathcal{T}_{h}\right)$.

3.2. Nonsymmetric formulations. Nonsymmetric DG approximations to the Poisson problem (and other selfadjoint PDEs) have received some interest in the literature. Such formulations use a nonsymmetric bilinear form that can be cast into the generic form

$$
a_{h}\left(v_{h}, w_{h}\right)=\int_{\Omega} \widehat{G}_{h}\left(v_{h}\right) \cdot G_{h}\left(w_{h}\right)+j_{h}^{\prime}\left(v_{h}, w_{h}\right),
$$

where $G_{h}$ is the discrete gradient considered above, whereas the discrete gradient $\widehat{G}_{h}$ and the stabilization bilinear form $j_{h}^{\prime}$ must satisfy the following design conditions:

(Ns1) Stability of the discrete gradient $\widehat{G}_{h}$ : there is $c$ s.t., for all $v_{h} \in V_{h}^{k}$,

$$
\left\|\widehat{G}_{h}\left(v_{h}\right)\right\|_{L^{2}(\Omega)^{d}} \leq c\left\|v_{h}\right\|_{\mathrm{DG}} .
$$

(Ns2) Strong convergence of the discrete gradient $\widehat{G}_{h}$ for smooth functions: for all $\varphi \in C_{c}^{\infty}(\Omega), \widehat{G}_{h}\left(\pi_{h} \varphi\right) \rightarrow \nabla \varphi$ in $L^{2}(\Omega)^{d}$.

(NS3) Stabilization: the bilinear form $j_{h}^{\prime}$ is symmetric and positive, and there is $c$ s.t. for all $v_{h} \in V_{h}^{k}, j_{h}^{\prime}\left(v_{h}, v_{h}\right) \leq c\left|v_{h}\right|_{\mathrm{J}, \mathcal{F}_{h},-1}^{2}$ (so that for all $\left(v_{h}, w_{h}\right) \in$ $\left.V_{h}^{k} \times V_{h}^{k}, j_{h}^{\prime}\left(v_{h}, w_{h}\right) \leq c\left|v_{h}\right|_{\mathrm{J}, \mathcal{F}_{h},-1}\left|w_{h}\right|_{\mathrm{J}, \mathcal{F}_{h},-1}\right)$.

(NS4) Coercivity: there is $\eta_{*}>0$ such that for all $v_{h} \in V_{h}^{k}$,

$$
a_{h}\left(v_{h}, v_{h}\right) \geq \eta_{*}\left\|v_{h}\right\|_{\mathrm{DG}}^{2} .
$$

Coercivity implies that the discrete problem (28) is well-posed. 
Under the above assumptions, the convergence of the sequence of discrete DG approximations can be proven. The proof, however, proceeds along a slightly different path with respect to the symmetric formulation.

Theorem 3.2. Let $\left\{u_{h}\right\}_{h \in \mathcal{H}}$ be the sequence of approximate solutions generated by solving the discrete problems (28) with the bilinear form $a_{h}$ given by (33) on the admissible meshes $\left\{\mathcal{T}_{h}\right\}_{h \in \mathcal{H}}$. Assume that the design conditions (N:11)-(N:4) hold. Then, as $\operatorname{size}\left(\mathcal{T}_{h}\right) \rightarrow 0, u_{h} \rightarrow u$ in $L^{2}(\Omega)$ and $\widehat{G}_{h}\left(u_{h}\right) \rightarrow \nabla u$ in $L^{2}(\Omega)^{d}$, where $u \in H_{0}^{1}(\Omega)$ is the unique solution to (18).

Proof. (i) Proceeding as before, it is inferred from (n\&4) that the sequence $\left\{u_{h}\right\}_{h \in \mathcal{H}}$ is bounded in the $\|\cdot\|_{\mathrm{DG}}$-norm, so that there exists $u \in H_{0}^{1}(\Omega)$ such that, up to a subsequence, $u_{h} \rightarrow u$ in $L^{2}(\Omega)$ and $G_{h}\left(u_{h}\right) \rightarrow \nabla u$ in $L^{2}(\Omega)^{d}$ as $\operatorname{size}\left(\mathcal{T}_{h}\right) \rightarrow 0$.

(ii) Strong convergence of $\widehat{G}_{h}\left(u_{h}\right)$. Let $\varphi \in C_{c}^{\infty}(\Omega)$. Observe that

$$
\begin{aligned}
\frac{1}{2}\left\|\widehat{G}_{h}\left(u_{h}\right)-\nabla u\right\|_{L^{2}(\Omega)^{d}}^{2} & \leq\left\|\widehat{G}_{h}\left(u_{h}\right)-\widehat{G}_{h}\left(\pi_{h} \varphi\right)\right\|_{L^{2}(\Omega)^{d}}^{2}+\left\|\widehat{G}_{h}\left(\pi_{h} \varphi\right)-\nabla u\right\|_{L^{2}(\Omega)^{d}}^{2} \\
& =T_{1}+T_{2} .
\end{aligned}
$$

Clearly, as $\operatorname{size}\left(\mathcal{T}_{h}\right) \rightarrow 0, T_{2} \rightarrow\|\nabla(\varphi-u)\|_{L^{2}(\Omega)^{d}}^{2}$ owing to $(\mathrm{N} 2)$. To bound $T_{1}$, use ( $\mathrm{N}$ 1) and ( $\mathrm{N}$ 4

$$
\begin{aligned}
T_{1}=\left\|\widehat{G}_{h}\left(u_{h}-\pi_{h} \varphi\right)\right\|_{L^{2}(\Omega)^{d}}^{2} & \leq \frac{c}{\eta_{*}} a_{h}\left(u_{h}-\pi_{h} \varphi, u_{h}-\pi_{h} \varphi\right) \\
& =\frac{c}{\eta_{*}}\left(\int_{\Omega} f\left(u_{h}-\pi_{h} \varphi\right)-a_{h}\left(\pi_{h} \varphi, u_{h}-\pi_{h} \varphi\right)\right) \\
& =\frac{c}{\eta_{*}}\left(T_{1,1}-T_{1,2}\right) .
\end{aligned}
$$

Clearly, as $\operatorname{size}\left(\mathcal{T}_{h}\right) \rightarrow 0, T_{1,1} \rightarrow \int_{\Omega} f(u-\varphi)$. Moreover, by definition,

$$
T_{1,2}=\int_{\Omega} \widehat{G}_{h}\left(\pi_{h} \varphi\right) \cdot G_{h}\left(u_{h}-\pi_{h} \varphi\right)+j_{h}^{\prime}\left(\pi_{h} \varphi, u_{h}-\pi_{h} \varphi\right) .
$$

Since $\widehat{G}_{h}\left(\pi_{h} \varphi\right)$ strongly converges to $\nabla \varphi$ in $L^{2}(\Omega)^{d}$ and $G_{h}\left(u_{h}-\pi_{h} \varphi\right)$ weakly converges to $\nabla(u-\varphi)$ in $L^{2}(\Omega)^{d}$, the first term in the right-hand side converges to $\int_{\Omega} \nabla \varphi \cdot \nabla(u-\varphi)$. The second term is bounded by $\left|\pi_{h} \varphi\right|_{\mathrm{J}, \mathcal{F}_{h},-1}\left|u_{h}-\pi_{h} \varphi\right|_{\mathrm{J}, \mathcal{F}_{h},-1}$ owing to $(\mathrm{N} 3$ ), and this bound converges to zero. Collecting the above bounds, it is inferred that

$$
\limsup \left\|\widehat{G}_{h}\left(u_{h}\right)-\nabla u\right\|_{L^{2}(\Omega)^{d}}^{2} \leq C\|u-\varphi\|_{H^{1}(\Omega)}^{2} .
$$

Using the density of $C_{c}^{\infty}(\Omega)$ in $H_{0}^{1}(\Omega)$, this upper bound can be made as small as desired. This proves the strong convergence of $\widehat{G}_{h}\left(u_{h}\right)$ to $\nabla u$ in $L^{2}(\Omega)^{d}$.

(iii) Identification of the limit and convergence of the whole sequence. Let $\varphi \in$ $C_{c}^{\infty}(\Omega)$. It is clear that as $\operatorname{size}\left(\mathcal{T}_{h}\right) \rightarrow 0, \int_{\Omega} f \pi_{h} \varphi \rightarrow \int_{\Omega} f \varphi$. Furthermore,

$$
a_{h}\left(u_{h}, \pi_{h} \varphi\right)=\int_{\Omega} \widehat{G}_{h}\left(u_{h}\right) \cdot G_{h}\left(\pi_{h} \varphi\right)+j_{h}^{\prime}\left(u_{h}, \pi_{h} \varphi\right)=T_{3}+T_{4} .
$$

Clearly, $T_{3} \rightarrow \int_{\Omega} \nabla u \cdot \nabla \varphi$. In addition, $T_{4}$ converges to zero since it is bounded by $\left|u_{h}\right|_{\mathrm{J}, \mathcal{F}_{h},-1}\left|\pi_{h} \varphi\right|_{\mathrm{J}, \mathcal{F}_{h},-1}$. As a result,

$$
\int_{\Omega} f \varphi \leftarrow \int_{\Omega} f \pi_{h} \varphi=a_{h}\left(u_{h}, \pi_{h} \varphi\right) \rightarrow \int_{\Omega} \nabla u \cdot \nabla \varphi
$$

The proof can now be concluded as in the symmetric case. 
Classical examples of the situation analyzed by Theorem 3.2 are the so-called Incomplete Interior Penalty method (IIPG) for which

$$
\widehat{G}_{h}\left(v_{h}\right)=\nabla_{h} v_{h},
$$

and the so-called Nonsymmetric Interior Penalty method (NIPG) for which

$$
\widehat{G}_{h}\left(v_{h}\right)=\nabla_{h} v_{h}+R_{h}\left(\llbracket v_{h} \rrbracket\right) .
$$

\section{The Stokes equations}

Let $f \in L^{r}(\Omega)^{d}$ with $r=\frac{2 d}{d+2}$ if $d \geq 3$ and $r>1$ if $d=2$. Let $\nu>0$. The components in the Cartesian basis $\left(e_{1}, \ldots, e_{d}\right)$ of $\mathbb{R}^{d}$ of a function, say $v$, with values in $\mathbb{R}^{d}$ will be denoted by $\left(v_{i}\right)_{1 \leq i \leq d}$. Implicit summation convention of repeated indices is adopted henceforth. Consider the Stokes equations

$$
\begin{cases}-\nu \Delta u_{i}+\partial_{i} p=f_{i}, & \text { in } \Omega, \quad i \in\{1, \ldots, d\}, \\ \partial_{i} u_{i}=0, & \text { in } \Omega, \\ u=0, & \text { on } \partial \Omega, \\ \int_{\Omega} p=0 . & \end{cases}
$$

The weak formulation of this system consists of finding $(u, p) \in H_{0}^{1}(\Omega)^{d} \times L_{0}^{2}(\Omega)$ s.t. for all $(v, q) \in H_{0}^{1}(\Omega)^{d} \times L_{0}^{2}(\Omega)$,

$$
\nu \int_{\Omega} \partial_{j} u_{i} \partial_{j} v_{i}-\int_{\Omega} p \partial_{i} v_{i}+\int_{\Omega} q \partial_{i} u_{i}=\int_{\Omega} f_{i} v_{i} .
$$

The well-posedness of the above problem is a classical result (see e.g. [16 and references therein).

To formulate a DG approximation, we consider for each component of the velocity the symmetric DG bilinear form $a_{h}$ defined by (21) and the stabilization bilinear form $j_{h}$ defined by (22). For the sake of simplicity, in particular with an eye towards ease of implementation, we will consider the case of equal-order polynomial interpolation for the velocity and for the pressure. Letting $k \geq 1$, we thus set

$$
U_{h} \stackrel{\text { def }}{=}\left[V_{h}^{k}\right]^{d}, \quad P_{h} \stackrel{\text { def }}{=} V_{h}^{k} / \mathbb{R}, \quad X_{h} \stackrel{\text { def }}{=} U_{h} \times P_{h} .
$$

For $\mathbb{R}^{d}$-valued functions such as velocities, the seminorm $|\cdot|_{\mathrm{J}, \mathcal{F}_{h},-1}$ and the norm $\|\cdot\|_{\mathrm{DG}}$ are defined as the square root of the sum of the squares of the corresponding seminorm or norm for all the components.

4.1. Discrete divergence operators. Define on $U_{h} \times P_{h}$ the bilinear form

$$
b_{h}\left(v_{h}, q_{h}\right) \stackrel{\text { def }}{=} \int_{\Omega} v_{h} \cdot \nabla_{h} q_{h}-\sum_{F \in \mathcal{F}_{h}^{i}} \int_{F} \nu_{F} \cdot\left\{v_{h}\right\} \llbracket \llbracket q_{h} \rrbracket .
$$

Integration by parts readily yields the following equivalent expression

$$
b_{h}\left(v_{h}, q_{h}\right)=-\int_{\Omega} q_{h} \nabla_{h} \cdot v_{h}+\sum_{F \in \mathcal{F}_{h}} \int_{F} \nu_{F} \cdot \llbracket v_{h} \rrbracket\left\{\left\{q_{h}\right\}\right\} .
$$

Here, $\nabla_{h}$. denotes the broken divergence operator acting elementwise. Furthermore, define on $P_{h} \times P_{h}$ the pressure stabilization bilinear form

$$
s_{h}\left(q_{h}, r_{h}\right) \stackrel{\text { def }}{=} \sum_{F \in \mathcal{F}_{h}^{i}} \gamma h_{F} \int_{F} \llbracket q_{h} \rrbracket \llbracket r_{h} \rrbracket .
$$


Here, $\gamma \in \mathbb{R}_{+}$is a penalty parameter. For simplicity, it will be taken equal to 1 in what follows. The basic stability result for the bilinear form $b_{h}$ is the following.

Lemma 4.1. There is $\beta>0$, depending on $\Omega, k$, and $\mathcal{P}$, such that

$$
\forall q_{h} \in P_{h}, \quad \beta\left\|q_{h}\right\|_{L^{2}(\Omega)} \leq \sup _{0 \neq v_{h} \in U_{h}} \frac{b_{h}\left(v_{h}, q_{h}\right)}{\left\|v_{h}\right\|_{\mathrm{DG}}}+\left|q_{h}\right|_{\mathrm{J}, \mathcal{F}_{h}^{i}, 1} .
$$

Proof. Let $q_{h} \in P_{h}$. Owing to a result by Nečas [32, there is $v \in H_{0}^{1}(\Omega)^{d}$ s.t. $\nabla \cdot v=q_{h}$ and $\|v\|_{H^{1}(\Omega)^{d}} \leq c_{\Omega}\left\|q_{h}\right\|_{L^{2}(\Omega)}$. Then,

$$
\begin{aligned}
\left\|q_{h}\right\|_{L^{2}(\Omega)}^{2}=\int_{\Omega} q_{h}(\nabla \cdot v) & =-\int_{\Omega} \nabla_{h} q_{h} \cdot v+\sum_{F \in \mathcal{F}_{h}^{i}} \int_{F} \llbracket q_{h} \rrbracket\{\{v\}] \cdot \nu_{F} \\
& =-\int_{\Omega} \nabla_{h} q_{h} \cdot \pi_{h}^{k} v+\sum_{F \in \mathcal{F}_{h}^{i}} \int_{F} \llbracket q_{h} \rrbracket\left\{\{v\} \cdot \nu_{F}\right. \\
& =-b_{h}\left(\pi_{h}^{k} v, q_{h}\right)+\sum_{F \in \mathcal{F}_{h}^{i}} \int_{F} \llbracket q_{h} \rrbracket\left\{\left\{v-\pi_{h}^{k} v\right\} \cdot \nu_{F}\right. \\
& =T_{1}+T_{2} .
\end{aligned}
$$

Since $\left\|\pi_{h}^{k} v\right\|_{\mathrm{DG}} \leq c_{k, \mathcal{P}}\|v\|_{H^{1}(\Omega)^{d}} \leq c_{\Omega, k, \mathcal{P}}\left\|q_{h}\right\|_{L^{2}(\Omega)}$, it is inferred that

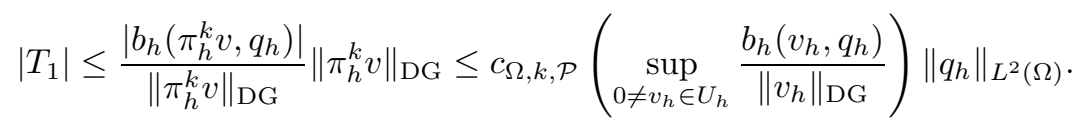

Similarly, using assumption (vi) in Definition 2.1] $\left|T_{2}\right| \leq c_{\Omega, k, \mathcal{P}}\left|q_{h}\right|_{\mathrm{J}, \mathcal{F}_{h}^{i}, 1}\left\|q_{h}\right\|_{L^{2}(\Omega)}$, whence the conclusion follows.

Remark 4.1. A more easily computable form for the pressure penalty term can be obtained by replacing $h_{F}$ with the quantity $\frac{\{|T|\}}{|F|}$ in (41) and redefining the seminorm $|\cdot|_{J, \mathcal{F}_{h}^{i}, 1}$ accordingly.

Recall the discrete gradient operators $G_{h}^{l}$ and $\mathcal{G}_{h}^{l}$ defined in 2.3 . For all $l \geq 0$, introduce now the discrete divergence operators $D_{h}^{l}: U_{h} \rightarrow V_{h}^{\max (k-1, l)}$ defined s.t.

$$
\forall v_{h} \in U_{h}, \quad D_{h}^{l}\left(v_{h}\right) \stackrel{\text { def }}{=} G_{h}^{l}\left(v_{h, j}\right) \cdot e_{j} .
$$

For $l \geq k$, the following integration by parts formula holds for all $\left(v_{h}, q_{h}\right) \in X_{h}$ :

$$
\int_{\Omega} q_{h} D_{h}^{l}\left(v_{h}\right)+\int_{\Omega} \mathcal{G}_{h}^{l}\left(q_{h}\right) \cdot v_{h}=0
$$

Moreover, it is easily seen that for $l \geq k$ and for all $\left(v_{h}, q_{h}\right) \in X_{h}$,

$$
b_{h}\left(v_{h}, q_{h}\right)=\int_{\Omega} v_{h} \cdot \mathcal{G}_{h}^{l}\left(q_{h}\right)=-\int_{\Omega} q_{h} D_{h}^{l}\left(v_{h}\right) .
$$

As before, superscripts will be dropped if $l=k$. 
4.2. Stability estimates and discrete well-posedness. Define for all $\left(\left(u_{h}, p_{h}\right)\right.$, $\left.\left(v_{h}, q_{h}\right)\right) \in X_{h} \times X_{h}$, the bilinear form

$$
l_{h}\left(\left(u_{h}, p_{h}\right),\left(v_{h}, q_{h}\right)\right) \stackrel{\text { def }}{=} \nu a_{h}\left(u_{h, i}, v_{h, i}\right)+b_{h}\left(v_{h}, p_{h}\right)-b_{h}\left(u_{h}, q_{h}\right)+s_{h}\left(p_{h}, q_{h}\right) .
$$

The discrete Stokes equations consist in finding $\left(u_{h}, p_{h}\right) \in X_{h}$ s.t.

$$
l_{h}\left(\left(u_{h}, p_{h}\right),\left(v_{h}, q_{h}\right)\right)=\int_{\Omega} f_{i} v_{h, i}, \quad \forall\left(v_{h}, q_{h}\right) \in X_{h} .
$$

Define the following norm:

$$
\left\|\left(v_{h}, q_{h}\right)\right\|_{\mathrm{S}}^{2} \stackrel{\text { def }}{=}\left\|v_{h}\right\|_{\mathrm{DG}}^{2}+\left|q_{h}\right|_{\mathrm{J}, \mathcal{F}_{h}^{i}, 1}^{2}+\left\|q_{h}\right\|_{L^{2}(\Omega)}^{2} .
$$

A direct consequence of (26) applied componentwise is the following result:

Lemma 4.2. Let $\alpha>0$ be as in Lemma 3.1. Then, the following holds:

$$
\forall\left(v_{h}, q_{h}\right) \in X_{h}, \quad \nu \alpha\left\|v_{h}\right\|_{\mathrm{DG}}^{2}+\left|q_{h}\right|_{\mathrm{J}, \mathcal{F}_{h}^{i}, 1}^{2} \leq l_{h}\left(\left(v_{h}, q_{h}\right),\left(v_{h}, q_{h}\right)\right) .
$$

Combining Lemmas 4.1 and 4.2 classically yields the following stability result.

Lemma 4.3. There is $c_{l}>0$ depending on $\nu, k, \mathcal{P}, \Omega$, and $\eta$ s.t.

$$
\forall\left(v_{h}, q_{h}\right) \in X_{h}, \quad c_{l}\left\|\left(v_{h}, q_{h}\right)\right\|_{\mathrm{S}} \leq \sup _{0 \neq\left(w_{h}, r_{h}\right) \in X_{h}} \frac{l_{h}\left(\left(v_{h}, q_{h}\right),\left(w_{h}, r_{h}\right)\right)}{\left\|\left(w_{h}, r_{h}\right)\right\|_{\mathrm{S}}} .
$$

A direct consequence of Lemma 4.3 is that for all $h \in \mathcal{H}$, the discrete problem (47) admits a unique solution $\left(u_{h}, p_{h}\right) \in X_{h}$.

4.3. Convergence analysis. In this section, we are now interested in the convergence of the sequence $\left\{\left(u_{h}, p_{h}\right)\right\}_{h \in \mathcal{H}}$ of solutions to the discrete Stokes equations (47) towards the unique solution $(u, p)$ of the continuous Stokes equations (37).

Theorem 4.1 (Convergence for Stokes equations). Let $\left\{\left(u_{h}, p_{h}\right)\right\}_{h \in \mathcal{H}}$ be the sequence of approximate solutions generated by solving the discrete problems (47) on the admissible meshes $\left\{\mathcal{T}_{h}\right\}_{h \in \mathcal{H}}$. Then, as $\operatorname{size}\left(\mathcal{T}_{h}\right) \rightarrow 0$,

$$
\begin{aligned}
u_{h} & \rightarrow u, \quad \text { in } L^{2}(\Omega)^{d}, \\
\nabla_{h} u_{h} & \rightarrow \nabla u, \quad \text { in } L^{2}(\Omega)^{d, d}, \\
\left|u_{h}\right|_{\mathrm{J}, \mathcal{F}_{h},-1} & \rightarrow 0, \\
p_{h} & \rightarrow p, \quad \text { in } L^{2}(\Omega), \\
\left|p_{h}\right|_{\mathrm{J}, \mathcal{F}_{h}^{i}, 1} & \rightarrow 0,
\end{aligned}
$$

where $(u, p) \in H_{0}^{1}(\Omega) \times L_{0}^{2}(\Omega)$ is the unique solution to (37).

Proof. (i) A priori estimates. Owing to the inf-sup condition (50), the assumption on $f$, and the discrete Sobolev embedding, the sequence $\left\{\left(u_{h}, p_{h}\right)\right\}_{h \in \mathcal{H}}$ is bounded in the $\|\cdot\|_{\mathrm{S}}$-norm. Hence, up to a subsequence, there is $(u, p) \in H_{0}^{1}(\Omega) \times L_{0}^{2}(\Omega)$ s.t. $u_{h} \rightarrow u$ strongly in $L^{2}(\Omega)^{d}, G_{h}\left(u_{h, i}\right) \rightarrow \nabla u_{i}$ weakly in $L^{2}(\Omega)^{d}$ for all $i \in\{1, \ldots, d\}$, and $p_{h} \rightarrow p$ weakly in $L^{2}(\Omega)$.

(ii) Identification of the limit and convergence of the whole sequence. Let $\varphi \in$ $C_{c}^{\infty}(\Omega)^{d}$. Testing with $\left(\pi_{h} \varphi, 0\right)$ yields

$$
\nu a_{h}\left(u_{h, i}, \pi_{h} \varphi_{i}\right)+b_{h}\left(\pi_{h} \varphi, p_{h}\right)=\int_{\Omega} f_{i} \pi_{h} \varphi_{i} .
$$


Clearly, as $\operatorname{size}\left(\mathcal{T}_{h}\right) \rightarrow 0$, the right-hand side tends to $\int_{\Omega} f_{i} \varphi_{i}$. Furthermore, proceeding as for the Poisson problem yields that the first term in the left-hand side converges to $\nu \int_{\Omega} \partial_{j} u_{i} \partial_{j} \varphi_{i}$. Consider now the second term and observe using (45) that $b_{h}\left(\pi_{h} \varphi, p_{h}\right)=-\int_{\Omega} p_{h} D_{h}\left(\pi_{h} \varphi\right)$. Owing to the weak convergence of $\left\{p_{h}\right\}_{h \in \mathcal{H}}$ to $p$ in $L^{2}(\Omega)$ and the strong convergence of $\left\{D_{h}\left(\pi_{h} \varphi\right)\right\}_{h \in \mathcal{H}}$ to $\nabla \cdot \varphi$ in $L^{2}(\Omega)$, $b_{h}\left(\pi_{h} \varphi, p_{h}\right)$ tends to $-\int_{\Omega} p(\nabla \cdot \varphi)$. As a result,

$$
\nu \int_{\Omega} \partial_{j} u_{i} \partial_{j} \varphi_{i}-\int_{\Omega} p \partial_{j} \varphi_{j}=\int_{\Omega} f_{i} \varphi_{i}
$$

Let now $\psi \in C_{c}^{\infty}(\Omega) / \mathbb{R}$. Testing with $\left(0, \pi_{h} \psi\right)$ yields

$$
-b_{h}\left(u_{h}, \pi_{h} \psi\right)+s_{h}\left(p_{h}, \pi_{h} \psi\right)=0 .
$$

Clearly, $-b_{h}\left(u_{h}, \pi_{h} \psi\right)=\int_{\Omega} \pi_{h} \psi D_{h}\left(u_{h}\right)$ tends to $\int_{\Omega} \psi(\nabla \cdot u)$ since $\left\{D_{h}\left(u_{h}\right)\right\}_{h \in \mathcal{H}}$ weakly converges to $\nabla \cdot u$ in $L^{2}(\Omega)$ and $\left\{\pi_{h} \psi\right\}_{h \in \mathcal{H}}$ strongly converges to $\psi$ in $L^{2}(\Omega)$. Furthermore, $s_{h}\left(p_{h}, \pi_{h} \psi\right)$ tends to zero since $\left|s_{h}\left(p_{h}, \pi_{h} \psi\right)\right| \leq\left|p_{h}\right|_{\mathrm{J}, \mathcal{F}_{h}^{i}, 1}\left|\pi_{h} \psi\right|_{\mathrm{J}, \mathcal{F}_{h}^{i}, 1} \leq$ $C\left|\pi_{h} \psi\right|_{\mathrm{J}, \mathcal{F}_{h}^{i}, 1}$, and this upper bound tends to zero. Hence,

$$
\int_{\Omega} \psi \partial_{j} u_{j}=0
$$

By density of $C_{c}^{\infty}(\Omega)^{d} \times C_{c}^{\infty}(\Omega) / \mathbb{R}$ in $H_{0}^{1}(\Omega)^{d} \times L_{0}^{2}(\Omega)$, this shows that $(u, p)$ solves the Stokes equations (37). Since the solution to this problem is unique, the whole sequence $\left\{\left(u_{h}, p_{h}\right)\right\}_{h \in \mathcal{H}}$ converges.

(iii) Strong convergence of the velocity gradient and convergence of velocity and pressure jumps. Observe that

$$
\begin{aligned}
\int_{\Omega} f_{i} u_{h, i}=l_{h}\left(\left(u_{h}, p_{h}\right),\left(u_{h}, p_{h}\right)\right) & \geq \nu a_{h}\left(u_{h, i}, u_{h, i}\right)+s_{h}\left(p_{h}, p_{h}\right) \\
& \geq \nu a_{h}\left(u_{h, i}, u_{h, i}\right) \geq \sum_{i=1}^{d} \nu\left\|G_{h}\left(u_{h, i}\right)\right\|_{L^{2}(\Omega)^{d}}^{2} .
\end{aligned}
$$

Thus,

$$
\limsup \sum_{i=1}^{d} \nu\left\|G_{h}\left(u_{h, i}\right)\right\|_{L^{2}(\Omega)^{d}}^{2} \leq \limsup \int_{\Omega} f_{i} u_{h, i}=\int_{\Omega} f_{i} u_{i}=\nu\|\nabla u\|_{L^{2}(\Omega)^{d, d}}^{2} .
$$

Proceeding as for the Poisson problem, it is inferred that $G_{h}\left(u_{h, i}\right) \rightarrow \nabla u_{i}$ for all $i \in\{1, \ldots, d\}$ in $L^{2}(\Omega)^{d}$ and that $\left|u_{h}\right|_{\mathrm{J}, \mathcal{F}_{h},-1} \rightarrow 0$. Finally, since

$$
\left|p_{h}\right|_{\mathrm{J}, \mathcal{F}_{h}^{i}, 1}^{2}=b_{h}\left(u_{h}, p_{h}\right)=\int_{\Omega} f_{i} u_{h, i}-\nu a_{h}\left(u_{h, i}, u_{h, i}\right),
$$

it is inferred that $\left|p_{h}\right|_{\mathrm{J}, \mathcal{F}_{h}^{i}, 1} \rightarrow 0$.

(iv) Strong convergence of the pressure. Using again the result by Nečas [32, let $v\left(p_{h}\right) \in H_{0}^{1}(\Omega)^{d}$ be s.t. $\nabla \cdot v\left(p_{h}\right)=p_{h}$ with $\left\|v\left(p_{h}\right)\right\|_{H^{1}(\Omega)^{d}} \leq c_{\Omega}\left\|p_{h}\right\|_{L^{2}(\Omega)}$ and set $v_{h}=\pi_{h}^{k} v\left(p_{h}\right)$. Then, proceeding as in the proof of Lemma 4.1 yields

$$
\begin{aligned}
\left\|p_{h}\right\|_{L^{2}(\Omega)}^{2} & \leq c_{\Omega, k, \mathcal{P}}\left|p_{h}\right|_{\mathrm{J}, \mathcal{F}_{h}^{i}, 1}\left\|p_{h}\right\|_{L^{2}(\Omega)}-b_{h}\left(v_{h}, p_{h}\right) \\
& \leq c_{\Omega, k, \mathcal{P}}\left|p_{h}\right|_{\mathrm{J}, \mathcal{F}_{h}^{i}, 1}\left\|p_{h}\right\|_{L^{2}(\Omega)}+\nu a_{h}\left(u_{h, i}, v_{h, i}\right)-\int_{\Omega} f_{i} v_{h, i}=T_{1}+T_{2}-T_{3} .
\end{aligned}
$$

Since $\left|p_{h}\right|_{J, \mathcal{F}_{h}^{i}, 1}$ tends to zero and $\left\|p_{h}\right\|_{L^{2}(\Omega)}$ is bounded, $T_{1}$ converges to zero. Furthermore, since the sequence $\left\{v_{h}\right\}_{h \in \mathcal{H}}$ is bounded in the $\|\cdot\|_{\mathrm{DG}}$-norm because 
$\left\|v_{h}\right\|_{\mathrm{DG}} \leq c_{k, \mathcal{P}}\left\|v\left(p_{h}\right)\right\|_{H^{1}(\Omega)^{d}} \leq c_{\Omega, k, \mathcal{P}}\left\|p_{h}\right\|_{L^{2}(\Omega)}$, there is $v \in H_{0}^{1}(\Omega)^{d}$ such that, up to a subsequence, $v_{h} \rightarrow v$ strongly in $L^{2}(\Omega)^{d}$ and $G_{h}\left(v_{h, i}\right) \rightarrow \nabla v_{i}$ weakly in $L^{2}(\Omega)^{d}$ for all $i \in\{1, \ldots, d\}$. Owing to the uniqueness of the limit in the distribution sense, it is inferred that $\nabla \cdot v=p$. Consider now the terms $T_{2}$ and $T_{3}$. It is clear that $T_{3} \rightarrow \int_{\Omega} f \cdot v$. Furthermore,

$$
T_{2}=\nu a_{h}\left(u_{h, i}, v_{h, i}\right)=\nu \int_{\Omega} G_{h}\left(u_{h, i}\right) \cdot G_{h}\left(v_{h, i}\right)+\nu j_{h}\left(u_{h, i}, v_{h, i}\right)=T_{2,1}+T_{2,2} .
$$

Owing to the strong convergence of $\left\{G_{h}\left(u_{h, i}\right)\right\}_{h \in \mathcal{H}}$ in $L^{2}(\Omega)^{d}$ and to the weak convergence of $\left\{G_{h}\left(v_{h, i}\right)\right\}_{h \in \mathcal{H}}$ in $L^{2}(\Omega)^{d}$, it is inferred that $T_{2,1} \rightarrow \nu \int_{\Omega} \partial_{j} u_{i} \partial_{j} v_{i}$. Moreover,

$$
\left|T_{2,2}\right| \leq c_{\nu, k, \mathcal{P}}\left|u_{h}\right|_{\mathrm{J}, \mathcal{F}_{h},-1}\left|v_{h}\right|_{\mathrm{J}, \mathcal{F}_{h},-1} \leq C\left|u_{h}\right|_{\mathrm{J}, \mathcal{F}_{h},-1},
$$

which converges to zero. Collecting the above estimates leads to

$$
\limsup \left\|p_{h}\right\|_{L^{2}(\Omega)}^{2} \leq \nu \int_{\Omega} \partial_{j} u_{i} \partial_{j} v_{i}-\int_{\Omega} f_{i} v_{i}=\int_{\Omega} p \partial_{j} v_{j}=\|p\|_{L^{2}(\Omega)}^{2},
$$

classically yielding the strong convergence of the pressure in $L^{2}(\Omega)$.

Remark 4.2. If the exact solution $(u, p)$ turns out to be more regular and belongs to the broken Sobolev space $H^{k+1}\left(\mathcal{T}_{h}\right)^{d} \times H^{k}\left(\mathcal{T}_{h}\right)$, optimal a priori error estimates of the form $\left\|\left(u-u_{h}, p-p_{h}\right)\right\|_{\mathrm{S}} \leq c_{u, p} \operatorname{size}\left(\mathcal{T}_{h}\right)^{k}$ can be established; see e.g. 11, 14, 19.

\section{The Steady incompressible Navier-Stokes equations}

In this section the space dimension is either 2 or 3. Let $f \in L^{r}(\Omega)^{d}$ with $r=\frac{6}{5}$ if $d=3$ and $r>1$ if $d=2$. Let $\nu>0$. Consider the steady incompressible Navier-Stokes equations in conservative form

$$
\begin{cases}-\nu \Delta u_{i}+\partial_{j}\left(u_{i} u_{j}\right)+\partial_{i} p=f_{i}, & \text { in } \Omega, \quad i \in\{1, \ldots, d\}, \\ \partial_{i} u_{i}=0, & \text { in } \Omega, \\ u=0, & \text { on } \partial \Omega, \\ \int_{\Omega} p=0 . & \end{cases}
$$

The weak formulation of this system consists in finding $(u, p) \in H_{0}^{1}(\Omega)^{d} \times L_{0}^{2}(\Omega)$ s.t. for all $(v, q) \in H_{0}^{1}(\Omega)^{d} \times L_{0}^{2}(\Omega)$,

$$
\nu \int_{\Omega} \partial_{j} u_{i} \partial_{j} v_{i}+\int_{\Omega} \partial_{j}\left(u_{i} u_{j}\right) v_{i}-\int_{\Omega} p \partial_{i} v_{i}+\int_{\Omega} q \partial_{i} u_{i}=\int_{\Omega} f_{i} v_{i} .
$$

The existence of a weak solution in the above sense, in two and three space dimensions, is a classical result; see, e.g., 33, 25. The uniqueness of the solution holds only under small data assumptions; see Remark 5.1 below.

5.1. Design of the convective trilinear form. We choose the same discrete spaces for the velocity and for the pressure as for the Stokes equations. To allow for some generality in the treatment of the convective term, we introduce two parameters $\alpha_{1}, \alpha_{2} \in\{0,1\}$ and rewrite the momentum equation in the NavierStokes equations as

$$
-\nu \Delta u_{i}+\partial_{j}\left(u_{i} u_{j}\right)-\alpha_{1} \frac{1}{2}\left(\partial_{j} u_{j}\right) u_{i}+\alpha_{2} \frac{1}{2} \partial_{i}\left(u_{j} u_{j}\right)+\partial_{i} \bar{p}=f_{i},
$$

with the modified pressure

$$
\bar{p} \stackrel{\text { def }}{=} p-\alpha_{2} \frac{1}{2}\left(u_{j} u_{j}\right) .
$$


The choice $\left(\alpha_{1}, \alpha_{2}\right)=(1,0)$ corresponds to Temam's device (see e.g. 33. to achieve stability. The choice $\left(\alpha_{1}, \alpha_{2}\right)=(0,1)$ has been hinted at in [10]; the modified pressure $\bar{p}$ differs from the Bernoulli pressure but the advantage is that the lefthand side of (58) is in divergence form, thereby lending itself to a conservative discretization. Define on $\left[H_{0}^{1}(\Omega)^{d}\right]^{3}$ the trilinear form

$$
t(w, u, v) \stackrel{\text { def }}{=} \int_{\Omega} \partial_{j}\left(w_{i} u_{j}\right) v_{i}-\alpha_{1} \frac{1}{2} \int_{\Omega}\left(\partial_{j} w_{j}\right) u_{i} v_{i}+\alpha_{2} \frac{1}{2} \int_{\Omega} \partial_{i}\left(w_{j} u_{j}\right) v_{i} .
$$

The discrete counterpart of the trilinear form $t$ is a trilinear form $t_{h}$ defined on $\left[U_{h}\right]^{3}$ and for which the following design conditions are relevant.

(T1) For all $v_{h} \in U_{h}$,

$$
t_{h}\left(v_{h}, v_{h}, v_{h}\right)=0 .
$$

(т2) There is $c_{t}$, depending on $k$ and $\mathcal{P}$, such that for all $\left(w_{h}, u_{h}, v_{h}\right) \in\left[U_{h}\right]^{3}$,

$$
t_{h}\left(w_{h}, u_{h}, v_{h}\right) \leq c_{t}\left\|w_{h}\right\|_{\mathrm{DG}}\left\|u_{h}\right\|_{\mathrm{DG}}\left\|v_{h}\right\|_{\mathrm{DG}} .
$$

(т3) Let $\left\{u_{h}\right\}_{h \in \mathcal{H}}$ be a sequence in $U_{h}$, bounded in the $\|\cdot\|_{\mathrm{DG}}$-norm. Then, for all $\varphi \in C_{c}^{\infty}(\Omega)^{d}$, as $\operatorname{size}\left(\mathcal{T}_{h}\right) \rightarrow 0$, up to a subsequence,

$$
t_{h}\left(u_{h}, u_{h}, \pi_{h} \varphi\right) \rightarrow t(u, u, \varphi),
$$

where $u \in H_{0}^{1}(\Omega)^{d}$ is given by Theorem 2.2 .

(т4) Assume furthermore that, for all $i \in\{1, \ldots, d\}, G_{h}\left(u_{h, i}\right) \rightarrow \nabla u_{i}$ strongly in $L^{2}(\Omega)^{d}$ and that $\left|u_{h}\right|_{\mathrm{J}, \mathcal{F}_{h},-1} \rightarrow 0$. Let $\left\{v_{h}\right\}_{h \in \mathcal{H}}$ be another sequence in $U_{h}$, bounded in the $\|\cdot\|_{\mathrm{DG}}$-norm. Then, as $\operatorname{size}\left(\mathcal{T}_{h}\right) \rightarrow 0$, up to a subsequence,

$$
t_{h}\left(u_{h}, u_{h}, v_{h}\right) \rightarrow t(u, u, v),
$$

where $v \in H_{0}^{1}(\Omega)^{d}$ is given by Theorem 2.2 .

5.2. Discrete well-posedness and basic stability estimates. The discrete problem consists in finding $\left(u_{h}, p_{h}\right) \in X_{h}$ s.t.

$$
l_{h}\left(\left(u_{h}, p_{h}\right),\left(v_{h}, q_{h}\right)\right)+t_{h}\left(u_{h}, u_{h}, v_{h}\right)=\int_{\Omega} f_{i} v_{h, i}, \quad \forall\left(v_{h}, q_{h}\right) \in X_{h},
$$

where the bilinear form $l_{h}$ associated with the Stokes equations is defined by (46). In this section, the discrete trilinear form $t_{h}$ is assumed to satisfy (Т1)-(т2) only.

Lemma 5.1 (A priori estimates). Let $\left(u_{h}, p_{h}\right) \in X_{h}$ and assume that $\left(u_{h}, p_{h}\right)$ solves (61). Then, the following a priori estimates hold:

$$
\begin{aligned}
(\nu \alpha)^{2}\left\|u_{h}\right\|_{\mathrm{DG}}^{2}+2 \alpha \nu\left|p_{h}\right|_{\mathrm{J}, \mathcal{F}_{h}^{i}, 1}^{2} & \leq \sigma_{r^{\prime}}^{2}\|f\|_{L^{r}(\Omega)^{d}}^{2}, \\
c_{l}\left\|\left(u_{h}, p_{h}\right)\right\|_{\mathrm{S}} & \leq \sigma_{r^{\prime}}\|f\|_{L^{r}(\Omega)^{d}}+c_{t}(\nu \alpha)^{-2}\left(\sigma_{r^{\prime}}\|f\|_{L^{r}(\Omega)^{d}}\right)^{2} .
\end{aligned}
$$

Proof. To prove (62), simply test (61) with $\left(u_{h}, p_{h}\right)$, observe that $t_{h}\left(u_{h}, u_{h}, u_{h}\right)=0$ owing to (T1) and use Lemma 4.2 for the linear part yielding

$$
\nu \alpha\left\|u_{h}\right\|_{\mathrm{DG}}^{2}+\left|p_{h}\right|_{\mathrm{J}, \mathcal{F}_{h}^{i}, 1}^{2} \leq \int_{\Omega} f_{i} u_{h, i} \leq \sigma_{r^{\prime}}\|f\|_{L^{r}(\Omega)^{d}}\left\|u_{h}\right\|_{\mathrm{DG}},
$$

whence (62) is easily deduced. To prove (63), use the inf-sup condition in Lemma 4.3 and assumption ( 2 2) to infer

$$
c_{l}\left\|\left(u_{h}, p_{h}\right)\right\|_{\mathrm{S}} \leq \sigma_{r^{\prime}}\|f\|_{L^{r}(\Omega)^{d}}+c_{t}\left\|u_{h}\right\|_{\mathrm{DG}}^{2},
$$

and conclude using (62). 
To prove the existence of a discrete solution, we use a topological degree argument; see, e.g., 20, 24 for the use of this argument in the convergence analysis of FV schemes and [13] for a general presentation.

Lemma 5.2. Let $V$ be a finite dimensional functional space equipped with a norm $\|\cdot\|_{V}$, let $\mu>0$, and let $\Psi: V \times[0,1] \rightarrow V$ satisfying the following assumptions:

(i) $\Psi$ is continuous.

(ii) $\Psi(\cdot, 0)$ is an affine function and the equation $\Psi(v, 0)=0$ has a solution $v \in V$ such that $\|v\|_{V}<\mu$.

(iii) For any $(v, \rho) \in V \times[0,1], \Psi(v, \rho)=0$ implies $\|v\|_{V} \neq \mu$.

Then, there exists $v \in V$ such that $\Psi(v, 1)=0$ and $\|v\|_{V}<\mu$.

Proposition 5.1. For all $h \in \mathcal{H}$, the discrete problem (61) admits at least one solution $\left(u_{h}, p_{h}\right) \in X_{h}$.

Proof. To apply Lemma 5.2 let $V=X_{h}$ and define the mapping $\Psi: X_{h} \times[0,1] \rightarrow X_{h}$ such that for $\left(u_{h}, p_{h}\right)$ given in $X_{h}$ and $\rho$ given in $[0,1],\left(\xi_{h}, \zeta_{h}\right) \stackrel{\text { def }}{=} \Psi\left(\left(u_{h}, p_{h}\right), \rho\right) \in$ $X_{h}$ is defined such that for all $\left(v_{h}, q_{h}\right) \in X_{h}$,

$$
\begin{aligned}
\left(\xi_{h}, v_{h}\right)_{L^{2}(\Omega)^{d}} & =l_{h}\left(\left(u_{h}, p_{h}\right),\left(v_{h}, 0\right)\right)+\rho t_{h}\left(u_{h}, u_{h}, v_{h}\right)-\int_{\Omega} f_{i} v_{h, i}, \\
\left(\zeta_{h}, q_{h}\right)_{L^{2}(\Omega)} & =l_{h}\left(\left(u_{h}, p_{h}\right),\left(0, q_{h}\right)\right) .
\end{aligned}
$$

Observing that $l_{h}$ is continuous on $X_{h} \times X_{h}$ for the $\|\cdot\|_{\mathrm{S}}$-norm, using (т2) and the equivalence of norms in finite dimension, it is inferred that $\Psi$ is continuous. Furthermore, point (ii) in Lemma 5.2 results from the a priori estimate for the Stokes equations. In addition, because of ( 1$)$, if $\left(u_{h}, p_{h}\right) \in X_{h}$ is such that $\Psi\left(\left(u_{h}, p_{h}\right), \rho\right)=0$ for some $\rho \in[0,1]$, then $\left(u_{h}, p_{h}\right)$ is bounded independently of $\rho$. This concludes the proof.

5.3. Convergence analysis. In this section, we are now interested in the convergence of a sequence $\left\{\left(u_{h}, p_{h}\right)\right\}_{h \in \mathcal{H}}$ of solutions to the discrete problem (61) towards a solution $(u, p)$ of the Navier-Stokes equations (57). The same convergence result can be established as for the Stokes equations. The only difference is that, because we do not make a smallness assumption on the data, there is no uniqueness result available at the continuous level, and thus only the convergence of subsequences (and not of the whole sequence) is obtained.

Theorem 5.1 (Convergence for Navier-Stokes equations). Let $\left\{\left(u_{h}, p_{h}\right)\right\}_{h \in \mathcal{H}}$ be a sequence of approximate solutions generated by solving the discrete problems (61) on the admissible meshes $\left\{\mathcal{T}_{h}\right\}_{h \in \mathcal{H}}$. Assume (т1)-(т3). Then, as $\operatorname{size}\left(\mathcal{T}_{h}\right) \rightarrow 0$, up to a subsequence,

$$
\begin{aligned}
u_{h} & \rightarrow u, \quad \text { in } L^{2}(\Omega)^{d}, \\
\nabla_{h} u_{h} & \rightarrow \nabla u, \quad \text { in } L^{2}(\Omega)^{d, d}, \\
\left|u_{h}\right|_{\mathrm{J}, \mathcal{F}_{h},-1} & \rightarrow 0, \\
p_{h} & \rightarrow \bar{p}, \quad \text { weakly in } L^{2}(\Omega), \\
\left|p_{h}\right|_{\mathrm{J}, \mathcal{F}_{h}^{i}, 1} & \rightarrow 0,
\end{aligned}
$$

where $\left(u, \bar{p}+\alpha_{2} \frac{1}{2}\left(u_{j} u_{j}\right)\right) \in H_{0}^{1}(\Omega) \times L_{0}^{2}(\Omega)$ is a solution to (57). Moreover, if ( $\left.\mathrm{T} 4\right)$ also holds, then $p_{h} \rightarrow \bar{p}$ in $L^{2}(\Omega)$. 
Proof. (i) Proceeding as for the Stokes equations, it is clear that there is $(u, \bar{p}) \in$ $H_{0}^{1}(\Omega) \times L_{0}^{2}(\Omega)$ s.t., up to a subsequence, $u_{h} \rightarrow u$ strongly in $L^{2}(\Omega)^{d}, G_{h}\left(u_{h, i}\right) \rightarrow$ $\nabla u_{i}$ weakly in $L^{2}(\Omega)^{d}$ for all $i \in\{1, \ldots, d\}$ and $p_{h} \rightarrow \bar{p}$ weakly in $L^{2}(\Omega)$.

(ii) Identification of the limit. Using ( т3) and proceeding as for the Stokes equations to treat the linear part, it is inferred that for all $\varphi \in C_{c}^{\infty}(\Omega)^{d}$,

$$
\nu \int_{\Omega} \partial_{j} u_{i} \partial_{j} \varphi_{i}+t(u, u, \varphi)-\int_{\Omega} \bar{p} \partial_{j} \varphi_{j}=\int_{\Omega} f_{i} \varphi_{i}
$$

and that for all $\psi \in C_{c}^{\infty}(\Omega) / \mathbb{R}$,

$$
\int_{\Omega} \psi \partial_{j} u_{j}=0
$$

Hence, $\left(u, \bar{p}+\alpha_{2} \frac{1}{2}\left(u_{j} u_{j}\right)\right)$ solves the incompressible Navier-Stokes equations.

(iii) Strong convergence of the velocity, the pressure, and their jumps. Proceeding as for the Stokes equations, (T1) yields the strong convergence of the broken velocity gradient in $L^{2}(\Omega)^{d}$ and the convergence to zero of the jump seminorms $\left|u_{h}\right|_{\mathrm{J}, \mathcal{F}_{h},-1}$ and $\left|p_{h}\right|_{\mathrm{J}, \mathcal{F}_{h}^{i}, 1}$. Furthermore, using again the velocity lifting of $p_{h}$ yields

$$
\begin{aligned}
\left\|p_{h}\right\|_{L^{2}(\Omega)}^{2} & \leq c_{\Omega, k, \mathcal{P}}\left|p_{h}\right|_{\mathrm{J}, \mathcal{F}_{h}^{i}, 1}\left\|p_{h}\right\|_{L^{2}(\Omega)}+\nu a_{h}\left(u_{h, i}, v_{h, i}\right)+t_{h}\left(u_{h}, u_{h}, v_{h}\right)-\int_{\Omega} f_{i} v_{h, i} \\
& =T_{1}+T_{2}+T_{3}-T_{4} .
\end{aligned}
$$

The convergence of $T_{1}, T_{2}$, and $T_{4}$ is treated the same as for the Stokes equations, while the convergence of $T_{3}$ results from assumption (T4). As a result,

$$
\begin{aligned}
\limsup \left\|p_{h}\right\|_{L^{2}(\Omega)}^{2} & \leq \nu \int_{\Omega} \partial_{j} u_{i} \partial_{j} v_{i}+t(u, u, v)-\int_{\Omega} f_{i} v_{i} \\
& =\int_{\Omega} p\left(\partial_{i} v_{i}\right)+\alpha_{2} \frac{1}{2} \int_{\Omega} \partial_{i}\left(u_{j} u_{j}\right) v_{i}=\int_{\Omega} \bar{p}\left(\partial_{i} v_{i}\right)=\|\bar{p}\|_{L^{2}(\Omega)}^{2},
\end{aligned}
$$

concluding the proof.

Remark 5.1. Under a smallness condition of the form

$$
c_{\Omega, k, \mathcal{P}} \nu^{-2}\|f\|_{L^{r}(\Omega)^{d}}<1,
$$

uniqueness of the weak solution of (57) classically holds, so that the conclusions (64) - (68) of Theorem 5.1 apply to the whole sequence $\left\{\left(u_{h}, p_{h}\right)\right\}_{h \in \mathcal{H}}$. Moreover, the convergence of the fixed-point iterative scheme

$$
l_{h}\left(\left(u_{h}^{k+1}, p_{h}^{k+1}\right),\left(v_{h}, q_{h}\right)\right)+t_{h}\left(u_{h}^{k}, u_{h}^{k+1}, v_{h}\right)=\int_{\Omega} f_{i} v_{h, i}, \quad \forall\left(v_{h}, q_{h}\right) \in X_{h},
$$

can be proven using standard arguments.

5.4. Examples. Define for $\left(w_{h}, u_{h}, v_{h}\right) \in\left[U_{h}\right]^{3}$,

$$
\begin{aligned}
t_{h}\left(w_{h}, u_{h}, v_{h}\right)= & \int_{\Omega}\left(w_{h} \cdot \nabla_{h} u_{h, i}\right) \cdot v_{h, i}-\sum_{F \in \mathcal{F}_{h}^{i}} \int_{F}\left\{w_{h} \rrbracket \cdot \nu_{F} \llbracket u_{h} \rrbracket \cdot\left\{\left\{v_{h}\right\}\right]\right. \\
& +\int_{\Omega} \frac{1}{2}\left(\nabla_{h} \cdot w_{h}\right)\left(u_{h} \cdot v_{h}\right)-\sum_{F \in \mathcal{F}_{h}} \int_{F} \llbracket w_{h} \rrbracket \cdot \nu_{F} \frac{1}{2}\left\{\left\{u_{h} \cdot v_{h}\right\}\right\} .
\end{aligned}
$$

This choice corresponds to $\left(\alpha_{1}, \alpha_{2}\right)=(1,0)$. The resulting DG method is not conservative, since it contains a source term proportional to the divergence of the discrete velocity (still converging to zero as the mesh is refined). 
Proposition 5.2. Let $t_{h}$ be defined by (69). Then, assumptions (T1)-(T4) hold.

Proof. The verification of (T1) is straightforward. Assumption (т2) results from the Sobolev embedding with $q=4$ and trace inequalities. To prove (т3) and (т4), observe first that for all $v_{h} \in U_{h}$,

$$
\begin{aligned}
t_{h}\left(u_{h}, u_{h}, v_{h}\right)= & \int_{\Omega} u_{h} \cdot \mathcal{G}_{h}^{2 k}\left(u_{h, i}\right) v_{h, i}+\frac{1}{4} \sum_{F \in \mathcal{F}_{h}^{i}} \int_{F} \llbracket u_{h, i} \rrbracket \nu_{F} \cdot \llbracket u_{h} \rrbracket \llbracket v_{h, i} \rrbracket \\
& +\int_{\Omega} D_{h}^{2 k}\left(u_{h}\right) \frac{1}{2} u_{h, i} v_{h, i}=T_{1}+T_{2}+T_{3} .
\end{aligned}
$$

To prove (T3), take $v_{h}=\pi_{h} \varphi$ with $\varphi \in C_{c}^{\infty}(\Omega)^{d}$. Owing to the discrete Sobolev embedding with $q=4$, the sequences $\left\{u_{h}\right\}_{h \in \mathcal{H}}$ and $\left\{\pi_{h} \varphi\right\}_{h \in \mathcal{H}}$ are bounded in $L^{4}(\Omega)^{d}$. Hence, Lebesgue's Dominated Convergence Theorem implies that, up to a subsequence, $u_{h} \pi_{h} \varphi_{i}$ converges to $u \varphi_{i}$ in $L^{2}(\Omega)^{d}$. In addition, $\left\{\mathcal{G}_{h}^{2 k}\left(u_{h, i}\right)\right\}_{h \in \mathcal{H}}$ weakly converges to $\nabla u_{i}$ in $L^{2}(\Omega)^{d}$. As a result, $T_{1}$ converges to $\int_{\Omega} u_{j}\left(\partial_{j} u_{i}\right) \varphi_{i}$. Similarly, $T_{3}$ converges to $\int_{\Omega} \frac{1}{2}\left(\partial_{j} u_{j}\right) u_{i} \varphi_{i}$. Furthermore, $T_{2} \rightarrow 0$ since $\left|u_{h}\right|_{\mathrm{J}, \mathcal{F}_{h},-1}$ is bounded and $\max _{F \in \mathcal{F}_{h}^{i}} h_{F}\left\|\llbracket \pi_{h} \varphi_{i} \rrbracket\right\|_{L^{\infty}(F)}$ converges to zero. Therefore, as $\operatorname{size}\left(\mathcal{T}_{h}\right) \rightarrow 0$,

$$
t_{h}\left(u_{h}, u_{h}, \pi_{h} \varphi\right) \rightarrow \int_{\Omega} u_{j}\left(\partial_{j} u_{i}\right) \varphi_{i}+\int_{\Omega} \frac{1}{2}\left(\partial_{j} u_{j}\right) u_{i} \varphi_{i}=\int_{\Omega}\left[\partial_{j}\left(u_{i} u_{j}\right)-\frac{1}{2}\left(\partial_{j} u_{j}\right) u_{i}\right] \varphi_{i},
$$

yielding the trilinear form $t$ with $\left(\alpha_{1}, \alpha_{2}\right)=(1,0)$. Assumption ( 44$)$ is proven similarly for the terms $T_{1}$ and $T_{3}$. To prove that $T_{2}$ converges to zero, observe that $\left|u_{h}\right|_{\mathrm{J}, \mathcal{F}_{h},-1}$ converges to zero and that $\max _{F \in \mathcal{F}_{h}^{i}} h_{F}\left\|\llbracket v_{h} \rrbracket\right\|_{L^{\infty}(F)} \leq c_{k, \mathcal{P}}\left|v_{h}\right|_{\mathrm{J}, \mathcal{F}_{h},-1}$ owing to an inverse inequality. This concludes the proof.

Now define for $\left(w_{h}, u_{h}, v_{h}\right) \in\left[U_{h}\right]^{3}$,

$$
\begin{aligned}
t_{h}\left(w_{h}, u_{h}, v_{h}\right)= & -\int_{\Omega} w_{h, i} u_{h} \cdot \nabla_{h} v_{h, i}+\sum_{F \in \mathcal{F}_{h}^{i}} \int_{F} \nu_{F} \cdot\left\{\left\{u_{h}\right\}\right\}\left\{\left\{w_{h, i}\right\} \llbracket \llbracket v_{h, i} \rrbracket\right. \\
& +\int_{\Omega} \frac{1}{2} v_{h} \cdot \nabla_{h}\left(u_{h, i} w_{h, i}\right)-\sum_{F \in \mathcal{F}_{h}^{i}} \int_{F} \nu_{F} \cdot\left\{\left\{v_{h}\right\}\right\} \frac{1}{2} \llbracket u_{h, i} w_{h, i} \rrbracket .
\end{aligned}
$$

This choice corresponds to $\left(\alpha_{1}, \alpha_{2}\right)=(0,1)$. The salient feature of the resulting DG method is that it is locally conservative.

Proposition 5.3. Let $t_{h}$ be defined by (70). Then, assumptions (T1)-(T4) hold.

Proof. Assumptions (T1)-(T2) can be readily verified. To prove (T3) and (T4), proceed as in the previous proof by observing that for all $v_{h} \in U_{h}$,

$$
\begin{aligned}
t_{h}\left(u_{h}, u_{h}, v_{h}\right)= & -\int_{\Omega} u_{h, i} u_{h} \cdot \mathcal{G}_{h}^{2 k}\left(v_{h, i}\right)-\frac{1}{4} \sum_{F \in \mathcal{F}_{h}^{i}} \int_{F} \nu_{F} \cdot \llbracket u_{h} \rrbracket \llbracket u_{h, i} \rrbracket \llbracket v_{h, i} \rrbracket \\
& -\int_{\Omega} \frac{1}{2} u_{h, i} u_{h, i} D_{h}^{2 k}\left(v_{h}\right) .
\end{aligned}
$$

In particular, for all $\varphi \in C_{c}^{\infty}(\Omega)^{d}$, as $\operatorname{size}\left(\mathcal{T}_{h}\right) \rightarrow 0$,

$$
t_{h}\left(u_{h}, u_{h}, \pi_{h} \varphi\right) \rightarrow \int_{\Omega}\left[\partial_{j}\left(u_{i} u_{j}\right)+\frac{1}{2} \partial_{i}\left(u_{j} u_{j}\right)\right] \varphi_{i},
$$

yielding the trilinear form $t$ with $\left(\alpha_{1}, \alpha_{2}\right)=(0,1)$. 
TABLE 1. Convergence results for the trilinear form defined by

(69). We have set $e_{h}=\left(e_{h, u}, e_{h, p}\right) \stackrel{\text { def }}{=}\left(u-u_{h}, p-p_{h}\right)$.

\begin{tabular}{|c|c|cccccc|}
\hline mesh & $h$ & $\left\|e_{h, u}\right\|_{L^{2}(\Omega) d^{d}}$ & order & $\left\|e_{h, p}\right\|_{L^{2}(\Omega)}$ & order & $\left\|e_{h}\right\|_{\mathrm{S}}$ & order \\
\hline 1 & $5.00 e-1$ & $8.87 e-01$ & - & $1.62 e+00$ & - & $1.19 e+01$ & - \\
2 & $2.50 e-1$ & $2.39 e-01$ & 1.89 & $6.11 e-01$ & 1.41 & $7.26 e+00$ & 0.71 \\
3 & $1.25 e-1$ & $5.94 e-02$ & 2.01 & $2.01 e-01$ & 1.60 & $3.68 e+00$ & 0.98 \\
4 & $6.25 e-2$ & $1.59 e-02$ & 1.90 & $7.40 e-02$ & 1.44 & $1.85 e+00$ & 0.99 \\
5 & $3.12 e-2$ & $4.17 e-03$ & 1.93 & $3.14 e-02$ & 1.23 & $9.25 e-01$ & 1.00 \\
\hline
\end{tabular}

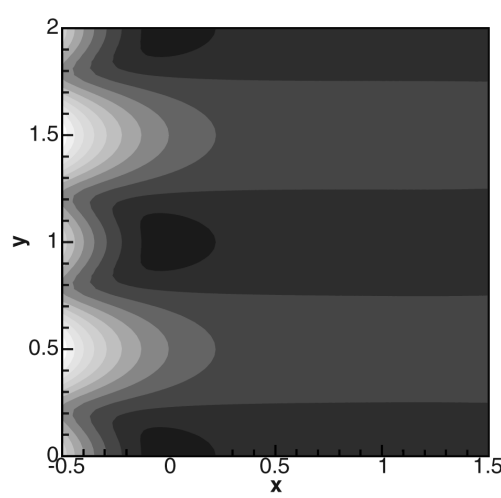

(a) Velocity module

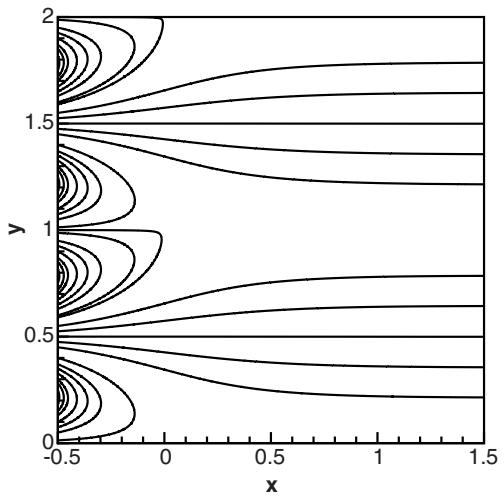

(b) Stream lines

FiguRE 2. Plot of Kovasznay's solution for $k=1$ and mesh 5 .

Remark 5.2. Upwinding can be introduced in the discrete trilinear forms $t_{h}$ defined by (69) or (70) by adding a term of the form

$$
\sum_{F \in \mathcal{F}_{h}^{i}} \theta_{F} \int_{F}\left|\left\{\left\{w_{h}\right\}\right] \cdot \nu_{F}\right| \llbracket u_{h} \rrbracket \cdot \llbracket v_{h} \rrbracket,
$$

and replacing the design assumption ( $\mathrm{T} 1)$ by the requirement that $t_{h}$ be nonnegative, which is sufficient to derive all the necessary a priori estimates and the convergence result of Theorem [5.1. Here, the parameter $\theta_{F} \in[0,1]$ depends on the local Péclet number.

5.5. Numerical experiment. To verify the asymptotic convergence properties of the method defined by (69), we have considered the analytical solution proposed in [28] on the square domain $\Omega \stackrel{\text { def }}{=}(-0.5,1.5) \times(0,2)$,

$$
u_{1}=1-e^{-\pi x_{2}} \cos \left(2 \pi x_{2}\right), \quad u_{2}=-\frac{1}{2} e^{\pi x_{1}} \sin \left(2 \pi x_{2}\right), \quad p=-\frac{1}{2} e^{\pi x_{1}} \cos \left(2 \pi x_{2}\right)-\widetilde{p},
$$

where $\tilde{p} \stackrel{\text { def }}{=} \frac{1}{\mathrm{~m}_{\Omega}} \int_{\Omega}-\frac{1}{2} e^{\pi x_{1}} \cos \left(2 \pi x_{2}\right) \simeq-0.920735694$ ensures zero-mean for the pressure, $\nu=\frac{1}{3 \pi}$, and $f=0$. The example was run on a family of uniformly refined triangular meshes with mesh sizes ranging from 0.5 down to 0.03125 , labeled with progressive numbers from 1 to 5 in Table 1 . The nonlinear problem was solved by the exact Newton algorithm with tolerance set to $10^{-6}$; the linear systems 
were solved using the direct solver available in PETSc. According to Table 1, the method converges with optimal order in the energy norm defined by (48).

\section{Discrete functional analysis in DG SPACES}

Let $1 \leq p<+\infty$ and let $k \geq 1$ be an integer. Equip the DG finite element space $V_{h}^{k}$ defined by (4) with the norm

$$
\left\|v_{h}\right\|_{\mathrm{DG}, p}^{p} \stackrel{\text { def }}{=} \sum_{T \in \mathcal{T}_{h}} \int_{T}\left|\nabla v_{h}\right|_{\ell^{p}}^{p}+\sum_{F \in \mathcal{F}_{h}} \frac{1}{h_{F}^{p-1}} \int_{F}\left|\llbracket v_{h} \rrbracket\right|^{p},
$$

where $|\cdot|_{\ell^{p}}$ denotes the $\ell^{p}$-norm in $\mathbb{R}^{d}$ so that $\left|\nabla v_{h}\right|_{\ell^{p}}^{p}=\sum_{i=1}^{d}\left|\partial_{i} v_{h}\right|^{p}$ (the Euclidian norm in $\mathbb{R}^{d}$ can also be used). Recall that $\Omega$ is an open bounded connected subset of $\mathbb{R}^{d}(d>1)$ whose boundary is a finite union of parts of hyperplanes. In this section, the mesh family $\left\{\mathcal{T}_{h}\right\}_{h \in \mathcal{H}}$ used to build the DG spaces is assumed to satisfy only assumptions (i)-(iv) in Definition 2.1.

The material contained in this section, which is closely inspired from that derived in 22 for discrete spaces of piecewise constant functions, deals with the extension to DG spaces of two key results of functional analysis, namely Sobolev embeddings and compactness criteria in $L^{p}(\Omega)$. These results are presented here in a non-Hilbertian setting which is more general than that needed to analyze the Navier-Stokes equations. We have made this choice because the results below are of independent interest to analyze other nonlinear problems; see also 9 . We also observe that we deal here with functional analysis in DG spaces and not in broken Sobolev spaces.

Lemma 6.1. For all $1 \leq s<t<+\infty$, the following holds for all $v_{h} \in V_{h}^{k}$,

$$
\left\|v_{h}\right\|_{\mathrm{DG}, s} \leq c_{d, \varrho_{1},|\Omega|, s, t}\left\|v_{h}\right\|_{\mathrm{DG}, t} .
$$

Proof. Observing that for all $x \in \mathbb{R}^{d},|x|_{\ell^{s}} \leq d^{\frac{1}{s}-\frac{1}{t}}|x|_{\ell^{t}}$ and using Hölder's inequality with $\pi=\frac{t}{s}>1$ and $\pi^{\prime}=\frac{\pi}{\pi-1}$ yields

$$
\begin{aligned}
\left\|v_{h}\right\|_{\mathrm{DG}, s}^{s}= & \sum_{T \in \mathcal{T}_{h}} \int_{T}\left|\nabla v_{h}\right|_{\ell^{s}}^{s}+\sum_{F \in \mathcal{F}_{h}} \frac{1}{h_{F}^{s-1}} \int_{F}\left|\llbracket v_{h} \rrbracket\right|^{s} \\
\leq & \sum_{T \in \mathcal{T}_{h}} \int_{T} d \frac{1}{\pi^{\prime}}\left|\nabla v_{h}\right|_{\ell^{t}}^{s}+\sum_{F \in \mathcal{F}_{h}} \int_{F} h_{F}^{\frac{1}{\pi^{\prime}}} h_{F}^{\frac{1}{\pi}(1-t)}\left|\llbracket v_{h} \rrbracket\right|^{s} \\
\leq & \left(\sum_{T \in \mathcal{T}_{h}} d \int_{T} 1^{\pi^{\prime}}\right)^{\frac{1}{\pi^{\prime}}}\left(\sum_{T \in \mathcal{T}_{h}} \int_{T}\left|\nabla v_{h}\right|_{\ell^{t}}^{t}\right)^{\frac{1}{\pi}} \\
& +\left(\sum_{F \in \mathcal{F}_{h}} h_{F} \int_{F} 1^{\pi^{\prime}}\right)^{\frac{1}{\pi^{\prime}}}\left(\sum_{F \in \mathcal{F}_{h}} \frac{1}{h_{F}^{t-1}} \int_{F}\left|\llbracket v_{h} \rrbracket\right|^{t}\right)^{\frac{1}{\pi}} \\
\leq & \left(\left(d+\varrho_{1}\right)|\Omega|\right)^{\frac{1}{\pi^{\prime}}}\left\|v_{h}\right\|_{\mathrm{DG}, t}^{s},
\end{aligned}
$$

using (11), whence the conclusion follows.

Lemma 6.2. For $v \in L^{1}\left(\mathbb{R}^{d}\right)$, define

$$
\|v\|_{\mathrm{BV}}=\sum_{i=1}^{d} \sup \left\{\int_{\mathbb{R}^{d}} u \partial_{i} \varphi ; \varphi \in C_{c}^{\infty}\left(\mathbb{R}^{d}\right),\|\varphi\|_{L^{\infty}\left(\mathbb{R}^{d}\right)} \leq 1\right\}
$$


and set $\mathrm{BV}=\left\{v \in L^{1}\left(\mathbb{R}^{d}\right) ;\|v\|_{\mathrm{BV}}<+\infty\right\}$. Then, extending discrete functions in $V_{h}^{k}$ by zero outside $\Omega$, there holds $V_{h}^{k} \subset \mathrm{BV}$ and for all $1 \leq p<+\infty$,

$$
\forall v_{h} \in V_{h}^{k}, \quad\left\|v_{h}\right\|_{\mathrm{BV}} \leq c_{d, \varrho_{1},|\Omega|, p}\left\|v_{h}\right\|_{\mathrm{DG}, p} .
$$

Proof. Clearly, owing to Lemma 6.1, it suffices to prove (73) for $p=1$. Integrating by parts, it is clear that for all $v_{h} \in V_{h}^{k}$ and for all $\varphi \in C_{c}^{\infty}\left(\mathbb{R}^{d}\right)$ with $\|\varphi\|_{L^{\infty}\left(\mathbb{R}^{d}\right)} \leq 1$,

$$
\int_{\mathbb{R}^{d}} v_{h} \partial_{i} \varphi=-\int_{\mathbb{R}^{d}}\left(e_{i} \cdot \nabla_{h} v_{h}\right) \varphi+\sum_{F \in \mathcal{F}_{h}} \int_{F} e_{i} \cdot \nu_{F} \llbracket v_{h} \rrbracket \varphi \leq\left\|v_{h}\right\|_{\mathrm{DG}, 1} .
$$

Hence, $\left\|v_{h}\right\|_{\mathrm{BV}} \leq d\left\|v_{h}\right\|_{\mathrm{DG}, 1}$, completing the proof.

Remark 6.1. In this section we could have allowed the case $k=0$, although the derived results are not as interesting as for $k \geq 1$ because $\|\cdot\|_{\mathrm{DG}, p}$ is not the natural norm with which to equip the space $V_{h}^{0}$ when working with FV approximations to nonlinear second-order PDEs. Indeed, on $V_{h}^{0}$, the first term on the right-hand side of (71) (the broken gradient) drops out, and this entails that a length scale different from $h_{F}$ must be used for the jump term, thereby also requiring an additional (mild) assumption on the mesh family; see 22. for the analysis in this case.

Remark 6.2. The observation that the $\|\cdot\|_{\mathrm{DG}, 2}$-norm controls the BV-norm can also be found in [30] in the framework of linear elasticity.

\subsection{Discrete Sobolev embeddings.}

Theorem 6.1 (Discrete Sobolev embeddings). For all $q$ such that

(i) $1 \leq q \leq p^{*} \stackrel{\text { def }}{=} \frac{p d}{d-p}$ if $1 \leq p<d$,

(ii) $1 \leq q<+\infty$ if $d \leq p<+\infty$,

there is $\sigma_{q, p}$ such that

$$
\forall v_{h} \in V_{h}^{k}, \quad\left\|v_{h}\right\|_{L^{q}(\Omega)} \leq \sigma_{p, q}\left\|v_{h}\right\|_{\mathrm{DG}, p} .
$$

The constant $\sigma_{q, p}$ additionally depends on $k,|\Omega|$, and $\mathcal{P}$. In particular, for the choice $q=p$ which is always possible,

$$
\forall v_{h} \in V_{h}^{k}, \quad\left\|v_{h}\right\|_{L^{p}(\Omega)} \leq \sigma_{p, p}\left\|v_{h}\right\|_{\mathrm{DG}, p} .
$$

Proof. We follow L. Nirenberg's proof of Sobolev embeddings.

(i) The case $p=1$. Set $1^{*} \stackrel{\text { def }}{=} \frac{d}{d-1}$. Then, owing to a classical result (see, e.g. 22 for a proof), for all $v \in \mathrm{BV}$,

$$
\|v\|_{L^{1^{*}}\left(\mathbb{R}^{d}\right)} \leq \frac{1}{2 d}\|v\|_{\mathrm{BV}} .
$$

Extending discrete functions in $V_{h}^{k}$ by zero outside $\Omega$, Lemma 6.2 yields

$$
\left\|v_{h}\right\|_{L^{1^{*}\left(\mathbb{R}^{d}\right)}} \leq \frac{1}{2}\left\|v_{h}\right\|_{\mathrm{DG}, 1}
$$

i.e. , (74) for $p=1$ and $q=1^{*}$ with $\sigma_{1,1^{*}}=\frac{1}{2}$, and hence for all $1 \leq q \leq 1^{*}$ since $\Omega$ is bounded.

(ii) The case $1<p<d$. Set $\alpha=\frac{p(d-1)}{d-p}$ and observe that $\alpha>1$. Considering the function $\left|v_{h}\right|^{\alpha}$ (extended by zero outside $\Omega$ ) and proceeding as above yields

$$
2\left(\int_{\Omega}\left|v_{h}\right|^{p^{*}}\right)^{\frac{d-1}{d}} \leq\left.\left.\sum_{T \in \mathcal{T}_{h}} \int_{T}|\nabla| v_{h}\right|^{\alpha}\right|_{\ell^{1}}+\left.\sum_{F \in \mathcal{F}_{h}} \int_{F}|\llbracket| v_{h}\right|^{\alpha} \rrbracket \mid \equiv T_{1}+T_{2} .
$$


Observe that a.e. in each $T \in \mathcal{T}_{h},\left.\left.\left|\partial_{i}\right| v_{h}\right|^{\alpha}|=\alpha| v_{h}\right|^{\alpha-1}\left|\partial_{i} v_{h}\right|$ for all $i \in\{1, \ldots, d\}$ so that $\left.\left.|\nabla| v_{h}\right|^{\alpha}\right|_{\ell^{1}}=\alpha\left|v_{h}\right|^{\alpha-1}\left|\nabla v_{h}\right|_{\ell^{1}}$. Using Hölder's inequality with $p$ and $q=\frac{p}{p-1}$, the first term in (77) is bounded as

$$
\begin{aligned}
\left|T_{1}\right| & \leq \alpha\left(\sum_{T \in \mathcal{T}_{h}} \int_{T}\left|v_{h}\right|^{q(\alpha-1)}\right)^{\frac{1}{q}}\left(\sum_{T \in \mathcal{T}_{h}} \int_{T}\left|\nabla v_{h}\right|_{\ell^{1}}^{p}\right)^{\frac{1}{p}} \\
& \leq \alpha d^{\frac{p-1}{p}}\left(\int_{\Omega}\left|v_{h}\right|^{p^{*}}\right)^{\frac{1}{q}}\left(\sum_{T \in \mathcal{T}_{h}} \int_{T}\left|\nabla v_{h}\right|_{\ell^{p}}^{p}\right)^{\frac{1}{p}} .
\end{aligned}
$$

Furthermore, observing that $\left.|\llbracket| v_{h}\right|^{\alpha} \rrbracket\left|\leq 2 \alpha\left\{\left|v_{h}\right|^{\alpha-1}\right\}\right| \llbracket v_{h} \rrbracket \mid$ and using again Hölder's inequality, it is inferred that the second term in (77) is bounded as

$$
\begin{aligned}
\left|T_{2}\right| & \leq \alpha \sum_{T \in \mathcal{T}_{h}} \sum_{F \subset \partial T} \int_{F} h_{F}^{\frac{1}{q}}\left|v_{h \mid T}\right|^{\alpha-1} h_{F}^{-\frac{1}{q}}\left|\llbracket v_{h} \rrbracket\right| \\
& \leq \alpha\left(\sum_{T \in \mathcal{T}_{h}} \sum_{F \subset \partial T} \int_{F} h_{F}\left|v_{h \mid T}\right|^{p^{*}}\right)^{\frac{1}{q}}\left(\sum_{T \in \mathcal{T}_{h}} \sum_{F \subset \partial T} \frac{1}{h_{F}^{p-1}} \int_{F}\left|\llbracket v_{h} \rrbracket\right|^{p}\right)^{\frac{1}{p}} \\
& \leq \alpha 2^{\frac{1}{p}} \tau_{p^{*}, k}^{\frac{1}{q}}\left(\int_{\Omega}\left|v_{h}\right|^{p^{*}}\right)^{\frac{1}{q}}\left(\sum_{F \in \mathcal{F}_{h}} \frac{1}{h_{F}^{p-1}} \int_{F}\left|\llbracket v_{h} \rrbracket\right|^{p}\right)^{\frac{1}{p}},
\end{aligned}
$$

where for $s \in \mathbb{R}_{+}, \tau_{s, k}$ is the constant in the trace inequality

$$
\forall \zeta \in \mathbb{P}_{k}(T), \quad \sum_{F \subset \partial T} h_{F} \int_{F}|\zeta|^{s} \leq \tau_{s, k} \int_{T}|\zeta|^{s},
$$

valid uniformly for all $h \in \mathcal{H}$ and for all $T \in \mathcal{T}_{h}$. This leads to

$$
\begin{aligned}
2\left(\int_{\Omega}\left|v_{h}\right|^{p^{*}}\right)^{\frac{d-1}{d}} & \leq \alpha\left(d+2^{\frac{1}{p-1}} \tau_{p^{*}, k}\right)^{\frac{1}{q}}\left(\int_{\Omega}\left|v_{h}\right|^{p^{*}}\right)^{\frac{1}{q}}\left\|v_{h}\right\|_{\mathrm{DG}, p} \\
& \leq \alpha\left(d^{\frac{1}{q}}+2^{\frac{1}{p}} \tau_{p^{*}, k}^{\frac{1}{q}}\right)\left(\int_{\Omega}\left|v_{h}\right|^{p^{*}}\right)^{\frac{1}{q}}\left\|v_{h}\right\|_{\mathrm{DG}, p} .
\end{aligned}
$$

Observing that $\frac{d-1}{d}-\frac{1}{q}=\frac{1}{p^{*}}$ yields (74).

(iii) The case $d \leq p<+\infty$. Fix any $q_{1}$ such that $p<q_{1}<+\infty$ and set $p_{1}=\frac{d q_{1}}{d+q_{1}}$ so that $1<p_{1}<d$ and $p_{1}^{*}=q_{1}$. Then, owing to point (ii) in this proof, it is inferred that for all $v_{h} \in V_{h}^{k},\left\|v_{h}\right\|_{L^{q_{1}(\Omega)}} \leq \sigma_{p_{1}, q_{1}}\left\|v_{h}\right\|_{\mathrm{DG}, p_{1}}$, and the conclusion follows from Lemma 6.1 since $p_{1} \leq p$.

Remark 6.3. A trace inequality is used in the above proof when bounding $T_{2}$. This is why Theorem 6.1 is stated on DG spaces and not on broken Sobolev spaces. We restate that broken Sobolev spaces are not used in the present analysis.

6.2. Compactness. In this section we are interested in sequences $\left\{v_{h}\right\}_{h \in \mathcal{H}}$ in $V_{h}^{k}$ which are bounded in the $\|\cdot\|_{\mathrm{DG}}$-norm.

Theorem 6.2 (Compactness). Let $\left\{v_{h}\right\}_{h \in \mathcal{H}}$ be a sequence in $V_{h}^{k}$ and assume that

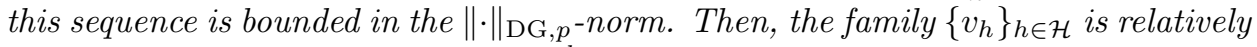
compact in $L^{p}(\Omega)$ (and also in $L^{p}\left(\mathbb{R}^{d}\right)$ taking $v_{h}=0$ outside $\Omega$ ). 
Proof. Extending the functions $v_{h}$ by zero outside $\Omega$ and observing that (see, e.g. 22]) for all $\xi \in \mathbb{R}^{d}$,

$$
\left\|v_{h}(\cdot+\xi)-v_{h}\right\|_{L^{1}\left(\mathbb{R}^{d}\right)} \leq|\xi|_{\ell^{1}}\left\|v_{h}\right\|_{\mathrm{BV}} \leq C|\xi|_{\ell^{1}},
$$

because of the boundedness of the sequence $\left\{v_{h}\right\}_{h \in \mathcal{H}}$ in the $\|\cdot\|_{\mathrm{DG}, p}$-norm (and hence in the BV-norm owing to Lemma 6.2), Kolmogorov's Compactness Criterion yields that the family $\left\{v_{h}\right\}_{h \in \mathcal{H}}$ is relatively compact in $L^{1}\left(\mathbb{R}^{d}\right)$. Owing to the Sobolev embedding (75), this sequence is also bounded in $L^{p}\left(\mathbb{R}^{d}\right)$; hence, it is also relatively compact in $L^{p}\left(\mathbb{R}^{d}\right)$. Finally, the relative compactness also holds in $L^{p}(\Omega)$ since the functions $v_{h}$ have been extended by zero outside $\Omega$.

Theorem 6.3 (Regularity of the limit). Let $1<p<+\infty$. Let $\left\{v_{h}\right\}_{h \in \mathcal{H}}$ be a sequence in $V_{h}^{k}$ and assume that this sequence is bounded in the $\|\cdot\|_{\mathrm{DG}, p}-$ norm. Assume that $\operatorname{size}\left(\mathcal{T}_{h}\right) \rightarrow 0$. Then, there exists $v \in W_{0}^{1, p}(\Omega)$ such that, up to a subsequence, $v_{h} \rightarrow v$ in $L^{p}(\Omega)$.

Proof. Owing to Theorem 6.2, there is $v \in L^{p}(\Omega)$ such that, up to a subsequence, $\left\{v_{h}\right\}_{h \in \mathcal{H}}$ converges to $v$ in $L^{p}(\Omega)$. It remains to prove that $v \in W_{0}^{1, p}(\Omega)$. To this purpose, we again extend the functions $v_{h}$ by zero outside $\Omega$ and we construct a discrete gradient converging, at least in the distribution sense over $\mathbb{R}^{d}$, to $\nabla v$.

(1) Consider the lifting operators $r_{F}^{0}$ and $R_{h}^{0}$ defined in $\$ 2.3$ and recall that the support of $r_{F}^{0}$ consists of the one or two mesh elements of which $F$ is a face. Hence,

$$
\begin{aligned}
\left\|R_{h}^{0}\left(\llbracket v_{h} \rrbracket\right)\right\|_{L^{p}(\Omega)^{d}}^{p} & =\sum_{T \in \mathcal{T}_{h}} \int_{T}\left|\sum_{F \subset \partial T} r_{F}^{0}\left(\llbracket v_{h} \rrbracket\right)\right|_{\ell^{p}}^{p} \\
& \leq \sum_{T \in \mathcal{T}_{h}} \int_{T} N_{\partial}^{p-1} \sum_{F \subset \partial T}\left|r_{F}^{0}\left(\llbracket v_{h} \rrbracket\right)\right|_{\ell^{p}}^{p} \\
& =N_{\partial}^{p-1} \sum_{F \in \mathcal{F}_{h}}\left\|r_{F}^{0}\left(\llbracket v_{h} \rrbracket\right)\right\|_{L^{p}(\Omega)^{d}}^{p} .
\end{aligned}
$$

Furthermore, setting for all $i \in\{1, \ldots, d\}, y_{h, i}=\left|r_{F, i}^{0}\left(\llbracket v_{h} \rrbracket\right)\right|^{p-2} r_{F, i}^{0}\left(\llbracket v_{h} \rrbracket\right)$, observing that $y_{h} \in\left[V_{h}^{0}\right]^{d}$ and using Hölder's inequality with $p$ and $q=\frac{p}{p-1}$ yields

$$
\begin{aligned}
\left\|r_{F}^{0}\left(\llbracket v_{h} \rrbracket\right)\right\|_{L^{p}(\Omega)^{d}}^{p} & =\int_{\Omega} y_{h} \cdot r_{F}^{0}\left(\llbracket v_{h} \rrbracket\right)=\int_{F}\left\{\left\{y_{h}\right\} \cdot \nu_{F} \llbracket v_{h} \rrbracket\right. \\
& \leq 2^{-\frac{1}{q}}\left(\sum_{T ; F \subset \partial T} h_{F} \int_{F}\left|y_{h \mid T} \cdot \nu_{F}\right|^{q}\right)^{\frac{1}{q}}\left(\frac{1}{h_{F}^{p-1}} \int_{F}\left|\llbracket v_{h} \rrbracket\right|^{p}\right)^{\frac{1}{p}} \\
& \leq 2^{-\frac{1}{q}}\left(\sum_{T ; F \subset \partial T} h_{F} d^{\frac{q}{p}} \int_{F}\left|r_{F}^{0}\left(\llbracket v_{h} \rrbracket\right)\right|_{\ell^{p}}^{p}\right)^{\frac{1}{q}}\left(\frac{1}{h_{F}^{p-1}} \int_{F}\left|\llbracket v_{h} \rrbracket\right|^{p}\right)^{\frac{1}{p}} \\
& \leq c_{d, p, k, \mathcal{P}}\left\|r_{F}^{0}\left(\llbracket v_{h} \rrbracket\right)\right\|_{L^{p}(\Omega)^{d}}^{\frac{p}{q}}\left(\frac{1}{h_{F}^{p-1}} \int_{F}\left|\llbracket v_{h} \rrbracket\right|^{p}\right)^{\frac{1}{p}} .
\end{aligned}
$$

Collecting the above bounds yields

$$
\left\|R_{h}^{0}\left(\llbracket v_{h} \rrbracket\right)\right\|_{L^{p}(\Omega)^{d}} \leq c_{d, p, k, \mathcal{P}}\left(\sum_{F \in \mathcal{F}_{h}} \frac{1}{h_{F}^{p-1}} \int_{F}\left|\llbracket v_{h} \rrbracket\right|^{p}\right)^{\frac{1}{p}} .
$$


Then, upon defining the approximate gradient $G_{h}^{0}\left(v_{h}\right)=\nabla_{h} v_{h}-R_{h}^{0}\left(\llbracket v_{h} \rrbracket\right) \in$ $\left[V_{h}^{k-1}\right]^{d}$ and extending it by zero outside $\Omega$, it is inferred that $\left\|G_{h}^{0}\left(v_{h}\right)\right\|_{L^{p}\left(\mathbb{R}^{d}\right)^{d}} \leq$ $c_{d, p, k, \mathcal{P}}\left\|v_{h}\right\|_{\mathrm{DG}, p}$. Hence, the sequence $\left\{G_{h}^{0}\left(v_{h}\right)\right\}_{h \in \mathcal{H}}$ is bounded in $L^{p}\left(\mathbb{R}^{d}\right)^{d}$, and thus since $p>1$, up to a subsequence, $G_{h}^{0}\left(v_{h}\right) \rightarrow w$ weakly in $L^{p}\left(\mathbb{R}^{d}\right)^{d}$.

(ii) Let $\varphi \in C_{c}^{\infty}\left(\mathbb{R}^{d}\right)^{d}$ and observe that

$$
\begin{aligned}
\int_{\mathbb{R}^{d}} G_{h}^{0}\left(v_{h}\right) \cdot \varphi= & -\int_{\mathbb{R}^{d}} v_{h}(\nabla \cdot \varphi)-\int_{\mathbb{R}^{d}} R_{h}^{0}\left(\llbracket v_{h} \rrbracket\right) \cdot\left(\varphi-\pi_{h}^{0} \varphi\right) \\
& \left.+\sum_{F \in \mathcal{F}_{h}} \int_{F}\left\{\varphi-\pi_{h}^{0} \varphi\right\}\right] \cdot \nu_{F} \llbracket v_{h} \rrbracket=T_{1}+T_{2}+T_{3} .
\end{aligned}
$$

Letting $\operatorname{size}\left(\mathcal{T}_{h}\right) \rightarrow 0$, we observe that $T_{1} \rightarrow-\int_{\mathbb{R}^{d}} v(\nabla \cdot \varphi)$ and that $T_{2} \rightarrow 0$ since $\left\|\varphi-\pi_{h}^{0} \varphi\right\|_{L^{q}\left(\mathbb{R}^{d}\right)^{d}} \rightarrow 0, q=\frac{p}{p-1}$, and $\left\|R_{h}^{0}\left(\llbracket v_{h} \rrbracket\right)\right\|_{L^{p}\left(\mathbb{R}^{d}\right)^{d}}$ is bounded. Furthermore, proceeding as usual,

$$
T_{3} \leq c_{\mathcal{P}}\left\|\varphi-\pi_{h}^{0} \varphi\right\|_{L^{\infty}\left(\mathbb{R}^{d}\right)^{d}}|\Omega|^{\frac{1}{q}}\left(\sum_{F \in \mathcal{F}_{h}} \frac{1}{h_{F}^{p-1}} \int_{F} \mid \llbracket v_{h} \rrbracket^{p}\right)^{\frac{1}{p}} \leq C\left\|\varphi-\pi_{h}^{0} \varphi\right\|_{L^{\infty}\left(\mathbb{R}^{d}\right)^{d}}
$$

whence it is inferred that $T_{3} \rightarrow 0$. As a result,

$$
\int_{\mathbb{R}^{d}} w \cdot \varphi=\lim _{\operatorname{size}\left(\mathcal{T}_{h}\right) \rightarrow 0} \int_{\mathbb{R}^{d}} G_{h}^{0}\left(v_{h}\right) \cdot \varphi=-\int_{\mathbb{R}^{d}} v(\nabla \cdot \varphi) .
$$

Hence, $w=\nabla v$ so that $v \in W^{1, p}(\Omega)$, and since $v$ is zero outside $\Omega, v \in W_{0}^{1, p}(\Omega)$.

Remark 6.4. For $p=2$, lifting operators using a higher polynomial degree $l \geq 1$ can also be considered as in the proof of Theorem 2.2. The difficulty for $p \neq 2$ is that the vector $y_{h}$ in the above proof is not necessarily polynomial-valued.

\section{ACKNOWLEDGMENT}

The authors are thankful to R. Eymard (University Paris-Est) for fruitful discussions.

\section{REFERENCES}

[1] D. N. Arnold, An interior penalty finite element method with discontinuous elements, SIAM J. Numer. Anal. 19 (1982), 742-760. MR664882 (83f:65173)

[2] D. N. Arnold, F. Brezzi, B. Cockburn, and D. Marini, Unified analysis of discontinuous Galerkin methods for elliptic problems, SIAM J. Numer. Anal. 39 (2002), no. 5, 1749-1779. MR1885715 (2002k:65183)

[3] F. Bassi, A. Crivellini, D. A. Di Pietro, and S. Rebay, An artificial compressibility flux for the discontinuous Galerkin solution of the incompressible Navier-Stokes equations, J. Comput. Phys. 218 (2006), no. 2, 794-815. MR.2269385 (2007f:76132)

[4] F. Bassi, S. Rebay, G. Mariotti, S. Pedinotti, and M. Savini, A high-order accurate discontinuous finite element method for inviscid and viscous turbomachinery flows, Proceedings of the $2^{\text {nd }}$ European Conference on Turbomachinery Fluid Dynamics and Thermodynamics (R. Decuypere and G. Dibelius, eds.), 1997, pp. 99-109.

[5] S. C. Brenner, Poincaré-Friedrichs inequalities for piecewise $H^{1}$ functions, SIAM J. Numer. Anal. 41 (2003), no. 1, 306-324. MR1974504 (2004d:65140)

[6] S. C. Brenner and L. R. Scott, The mathematical theory of finite element methods, Second ed., Texts in Applied Mathematics, vol. 15, Springer-Verlag, New York, 2002. MR 1894376 (2003a:65103)

[7] H. Brezis, Analyse fonctionnelle, 2005 ed., Masson, Paris, 1983. MR697382(85a:46001) 
[8] F. Brezzi, G. Manzini, D. Marini, P. Pietra, and A. Russo, Discontinuous Galerkin approximations for elliptic problems, Numer. Methods Partial Differential Equations 16 (2000), no. 4, 365-378. MR.1765651 (2001e:65178)

[9] A. Buffa and C. Ortner, Compact embeddings of broken Sobolev spaces and applications, IMA J. Numer. Anal. (2009), To appear.

[10] B. Cockburn, G. Kanschat, and D. Schötzau, A locally conservative LDG method for the incompressible Navier-Stokes equations, Math. Comp. 74 (2005), 1067-1095. MR2136994 (2006a:65157)

[11] B. Cockburn, G. Kanschat, D. Schötzau, and C. Schwab, Local Discontinuous Galerkin methods for the Stokes system, SIAM J. Numer. Anal. 40 (2002), no. 1, 319-343 (electronic). MR1921922 (2003g:65141)

[12] B. Cockburn and C.-W. Shu, The local discontinuous Galerkin finite element method for convection-diffusion systems, SIAM J. Numer. Anal. 35 (1998), 2440-2463. MR1655854 (99j:65163)

[13] K. Deimling, Nonlinear functional analysis, Springer-Verlag, Berlin, 1985. MR787404 (86j:47001)

[14] D. A. Di Pietro, Analysis of a discontinuous Galerkin approximation of the Stokes problem based on an artificial compressibility flux, Int. J. Numer. Methods Fluids 55 (2007), 793-813. MR2359551(2008i:76119)

[15] D. A. Di Pietro, A. Ern, and J.-L. Guermond, Discontinuous Galerkin methods for anisotropic semi-definite diffusion with advection, SIAM J. Numer. Anal. 46 (2008), no. 2, 805-831. MR.2383212 (2008k:65240)

[16] A. Ern and J.-L. Guermond, Theory and practice of finite elements, Applied Mathematical Sciences, vol. 159, Springer-Verlag, New York, NY, 2004. MR2050138 (2005d:65002)

[17] _ Discontinuous Galerkin methods for Friedrichs' systems. I. General theory, SIAM J. Numer. Anal. 44 (2006), no. 2, 753-778. MR2218968 (2007g:65107)

[18] _ Discontinuous Galerkin methods for Friedrichs' systems. II. Second-order elliptic PDEs, SIAM J. Numer. Anal. 44 (2006), no. 6, 2363-2388. MR.2272598 (2007k:65179)

[19] _ Discontinuous Galerkin methods for Friedrichs' systems. III. Multi-field theories with partial coercivity, SIAM J. Numer. Anal. 46 (2008), no. 2, 776-804. MR2383211 (2009b:65303)

[20] R. Eymard, Th. Gallouët, M. Ghilani, and R. Herbin, Error estimates for the approximate solutions of a nonlinear hyperbolic equation given by finite volume schemes, IMA J. Numer. Anal. 18 (1998), no. 4, 563-594. MR1681074 (2000b:65180)

[21] R. Eymard, Th. Gallouët, and R. Herbin, The finite volume method, Ph. Charlet and J.L. Lions eds, North Holland, 2000. MR.1804748 (2002e:65138)

$[22] \ldots$, Discretization schemes for heterogeneous and anisotropic diffusion problems on general nonconforming meshes, Available online as HAL report 00203269, January 2008, Submitted for publication.

[23] R. Eymard, Th. Gallouët, R. Herbin, and J.-C. Latché, Analysis tools for finite volume schemes, Acta Math. Univ. Comenian. (N.S.) 76 (2007), no. 1, 111-136. MR2331058 (2008e:65243)

[24] R. Eymard, R. Herbin, and J.-C. Latché, Convergence analysis of a colocated finite volume scheme for the incompressible Navier-Stokes equations on general $2 D$ or $3 D$ meshes, SIAM J. Numer. Anal. 45 (2007), no. 1, 1-36. MR2285842 (2008f:65182)

[25] V. Girault and P.-A. Raviart, Finite element methods for Navier-Stokes equations, Springer Series in Computational Mathematics, vol. 5, Springer-Verlag, Berlin, 1986, Theory and algorithms. MR851383 (88b:65129)

[26] V. Girault, B. Rivière, and M. F. Wheeler, A discontinuous Galerkin method with nonoverlapping domain decomposition for the Stokes and Navier-Stokes problems, Math. Comp. $\mathbf{7 4}$ (2005), no. 249, 53-84 (electronic). MR2085402 (2005f:65149)

[27] O. A. Karakashian and W. N. Jureidini, A nonconforming finite element method for the stationary Navier-Stokes equations, SIAM J. Numer. Anal. 35 (1998), no. 1, 93-120 (electronic). MR.1618436 (99d:65320)

[28] L. S. G. Kovasznay, Laminar flow behind a two-dimensional grid, Proc. Camb. Philos. Soc. 44 (1948), 58-62. MR0024282 (9:476d)

[29] A. Lasis and E. Süli, Poincaré-type inequalities for broken Sobolev spaces, Tech. Report 03/10, Oxford University Computing Laboratory, Oxford, England, 2003. 
[30] A. Lew, P. Neff, D. Sulsky, and M. Ortiz, Optimal BV estimates for a discontinuous Galerkin method for linear elasticity, AMRX Appl. Math. Res. Express 3 (2004), 73-106. MR2091832 (2005f:74034)

[31] I. Mozolevski, E. Süli, and P. R. Bösing, Discontinuous Galerkin finite element approximation of the two-dimensional Navier-Stokes equations in stream-function formulation, Comm. Numer. Methods Engrg. 23 (2007), no. 6, 447-459. MR.2329348 (2008c:76061)

[32] J. Nečas, Equations aux dérivées partielles, Presses de l'Université de Montréal, Montréal, Canada, 1965.

[33] R. Temam, Navier-Stokes Equations, revised ed., Studies in Mathematics and its Applications, vol. 2, North-Holland Publishing Co., Amsterdam, 1979, Theory and numerical analysis, With an appendix by F. Thomasset. MR603444 (82b:35133)

Institut Français du Pétrole, 1 \& 4, avenue du Bois-Préau, 92852 Rueil-Malmaison Cedex, France

E-mail address: daniele-antonio.di-pietro@ifp.fr

Université Paris-Est, CERMics, Ecole des Ponts, Champs sur Marne, 77455 Marne la Vallée Cedex 2, France

E-mail address: ern@cermics.enpc.fr 